\title{
PRODUCT FORMULA, TAILS AND INDEPENDENCE OF MULTIPLE STABLE INTEGRALS
}

\author{
Jan Rosiński and Gennady Samorodnitsky \\ University of Tennessee and Cornell University
}

\begin{abstract}
We give necessary and sufficient conditions for multiple integrals of arbitrary (not necessary equal) orders with respect to a symmetric stable random measure to be independent. This complements known results for multiple Wiener-Itô integrals. Similarly to Wiener-Itô integrals, the main tool in establishing independence is the product formula for multiple stochastic integrals. The role of Itô's isometry in the Gaussian case is replaced by the exact evaluation of the tail of stable integrals and their products.
\end{abstract}

\section{Introduction and Preliminaries.}

Stochastic independence is the basic notion in probability theory and, arguably, the one that distinguishes probability from other related areas of mathematics. Intuitively, "random objects" are independent when they have no "common causes". However, cancellations sometimes may occur. For example, if $X_{1}$ and $X_{2}$ are independent standard normal random variables then $Y_{1}=\left(X_{1}+X_{2}\right) / 2$ and $Y_{2}=$ $\left(X_{1}-X_{2}\right) / 2$ are independent even though they have "common causes": $X_{1}$ and $X_{2}$. To what extent is this "cancellation" phenomenon restricted to the Gaussian

1991 Mathematics Subject Classification. 60E07,60H05.

Key words and phrases. multiple stochastic integral, probability tails, stable distribution, random measure, product formula, independence, non-Gaussian chaos.

Jan Rosiński was partially supported by NSF grant DMS-97-04744 at University of Tennessee, Knoxville. Gennady Samorodnitsky was partially supported by NSF Grant DMS-97-04982 and NSA Grant MDA904-98-1-0041 at Cornell University. The authors acknowledge support provided by the Tennessee Science Alliance of a visit by G. Samorodnitsky to Knoxville when this paper was completed. 
world? It is natural to look at jointly infinitely divisible random variables without Gaussian components. They can be represented by stochastic integrals of deterministic functions with respect to an infinitely divisible random measure without Gaussian component. Urbanik (1967) showed that such integrals are independent if and only if the integrands have disjoint supports. Therefore, there are no common Poisson jumps in the sequence of independent stochastic integrals. In other words, Poissonian jumps do not seem to cancel.

How far does this "rigidity" of Poissonian jumps in comparison with cancellation flexibility of Gaussian random variables extend? It is interesting to look at multiple integrals with respect to Gaussian and infinitely divisible random measures. It has been proved by Üstünel and Zakai (1989) that for multiple Wiener-Itô integrals independence is equivalent to the $L^{2}$ orthogonality of one-dimensional sections of the two kernels, and so the two integrals may well be affected by the same randomness of the Gaussian random measure. On the other hand, this should not be the case for multiple integrals with respect to an infinitely divisible random measure without a Gaussian component if the Poissonian jumps did not cancel. A formal statement to this end was made by Privault (1996) who claimed that, for a particular class of compound Poisson random measures, independence is equivalent to disjoint supports of one-dimensional sections of the two kernels. Unfortunately, not only does the above paper deal with a very restricted class of infinitely divisible random measures, but there appears to be an error in the proof of the necessity part of the results (see Example 5.3).

In this paper we consider multiple integrals with respect to symmetric $\alpha$-stable random measures, $0<\alpha<2$, and show that in this case independence is indeed equivalent to disjoint supports of one-dimensional sections of the two kernels. It is interesting to note the difference in the approaches one takes to establish conditions for independence in the case of multiple Wiener-Itô integrals and in the case of multiple integrals with respect to a stable random measure. In the former case one uses $L^{2}$ theory, that is clearly unavailable in the heavy tailed stable case. In the 
latter case one uses probability tails of the multiple integrals.

We proceed with a more formal setup. Throughout this paper $(S, \sigma(\mathcal{S}))$ will denote a measurable space, where $\mathcal{S}$ is a $\delta$-ring of subsets of $S$ with the property that $S=\bigcup_{n} S_{n}$, for some sequence $\left\{S_{n}\right\} \subset \mathcal{S}$. Let $M$ be a symmetric infinitely divisible random measure without Gaussian component on $(S, \mathcal{S})$. That is, $\{M(A), A \in \mathcal{S}\}$ is a stochastic process such that (i) for every $A \in \mathcal{S}, M(A)$ is a symmetric infinitely divisible random variable without Gaussian component, (ii) for pairwise disjoint $A_{1}, A_{2}, \ldots$ in $\mathcal{S}$ the random variables $M\left(A_{1}\right), M\left(A_{2}\right), \ldots$ are independent (" $M$ is independently scattered"), and if $\cup_{n=1}^{\infty} A_{n} \in \mathcal{S}$ then $M\left(\cup_{n=1}^{\infty} A_{n}\right)=\sum_{n=1}^{\infty} M\left(A_{n}\right)$ a.s. (" $M$ is $\sigma$-additive").

The characteristic function of $M(A), A \in \mathcal{S}$, can be written in the form

$$
E \exp \{i \theta M(A)\}=\exp \left\{\int_{A} \int_{0}^{\infty}(1-\cos \theta x) \rho(d x, s) \lambda(d s)\right\},
$$

where $\lambda$ is a probability measure on $(S, \mathcal{S})$ and $\{\rho(\cdot, s), s \in S\}$ is a measurable family of Lévy measures on $(0, \infty)$, and

$$
\lambda\{s \in S: \rho((0, \infty), s)=0\}=0
$$

(see, e.g., Rajput and Rosiński (1989)). Condition (1.2) implies that $M(A)=0$ a.s. if and only if $\lambda(A)=0$, that is $\lambda$ is a control measure of $M$. Throughout this paper we will assume that $\lambda$ is atomless.

We will now describe the series expansion of $M$. For $s \in S$ let

$$
R(u, s)=\inf \{x>0: \rho((x, \infty), s) \leq u\}, \quad u>0
$$

Let $\left\{\epsilon_{i}, i \geq 1\right\},\left\{V_{i}, i \geq 1\right\}$ and $\left\{\Gamma_{i}, i \geq 1\right\}$ be independent sequences of random variables, the first one being a sequence of i.i.d. Rademacher random variables, the second one being a sequence of i.i.d. $S$-valued random variables with common distribution $\lambda$, while the last one being a sequence of arrival times of a Poisson process on $(0, \infty)$ with unit rate. Then, for every $A \in \mathcal{S}$, the series

$$
M_{0}(A)=\sum_{i=1}^{\infty} \epsilon_{i} R\left(\Gamma_{i}, V_{i}\right) \delta_{V_{i}}(A)
$$


converges a.s. and, $\left\{M_{0}(A), A \in \mathcal{S}\right\}={ }^{d}\{M(A), A \in \mathcal{S}\}$, where $=^{d}$ reads "equal in distribution" (see LePage (1980), Rosiński (1990)). Without loss of generality we will identify $M$ with its version $M_{0}$ given by (1.3), and we will write

$$
M(A)=\sum_{i=1}^{\infty} \xi_{i} \delta_{V_{i}}(A), \quad A \in \mathcal{S}
$$

where $\xi_{i}=\epsilon_{i} R\left(\Gamma_{i}, V_{i}\right)$. Let $f: S^{p} \rightarrow \mathbf{R}$ be a product measurable symmetric function (that is, $f$ is invariant under permutations of its arguments) and such that $f$ vanishes on the diagonals (that is, $f(\mathbf{s})=0$ if two or more of the coordinates of $\mathbf{s}$ coincide.) The multiple integral $I_{p}^{M}(f)=\int_{S^{p}} f d M^{\otimes p}$ can be defined on one hand as the integral with respect to the product random measure $M^{\otimes p}$ (see Kwapien and Woyczyński (1992)) and, on the other hand, it can equivalently be defined as a multilinear random form

$$
I_{p}^{M}(f)=\sum_{k_{1}, \ldots, k_{p}} \xi_{k_{1}} \cdots \xi_{k_{p}} f\left(V_{k_{1}}, \ldots, V_{k_{p}}\right)
$$

(see Kallenberg and Szulga (1989)). $I_{p}^{M}(f)$ exists if and only if

$$
\sum_{k_{1}, \ldots, k_{p}} \xi_{k_{1}}^{2} \cdots \xi_{k_{p}}^{2} f^{2}\left(V_{k_{1}}, \ldots, V_{k_{p}}\right)<\infty \quad \text { a.s. }
$$

Finally, we note that the multiple integral can be immediately extended to random integrands $F: \Omega \times S^{p} \rightarrow \mathbf{R}$ such that

(i) there is a $\sigma$-field $\mathcal{G}$ independent of the Rademacher sequence $\left\{\epsilon_{j}\right\}$ such that for every $\mathbf{s} \in S^{p}, F(\cdot, \mathbf{s})$ is measurable $\mathcal{G}$

(ii) for every $\omega \in \Omega, F(\omega, \cdot)$ is symmetric and vanishes on diagonals.

Indeed, conditions (i) and (ii) imply that

$$
I_{p}^{M}(F):=\sum_{k_{1}, \ldots, k_{p}} \xi_{k_{1}} \cdots \xi_{k_{p}} F\left(\cdot, V_{k_{1}}, \ldots, V_{k_{p}}\right)
$$

is a conditional Rademacher multilinear form which converges unconditionally a.s. if and only if

$$
\sum_{k_{1}, \ldots, k_{p}} \xi_{k_{1}}^{2} \cdots \xi_{k_{p}}^{2} F^{2}\left(\cdot, V_{k_{1}}, \ldots, V_{k_{p}}\right)<\infty \quad \text { a.s. }
$$


Hence (1.8) is a necessary and sufficient condition for the existence of $I_{p}^{M}(F)$ provided (i) and (ii) hold. Further, (1.8) is a sufficient condition of the existence of $I_{p}^{M}(F)$ for nonsymmetric $F$ 's, but it is not necessary. Clearly, $I_{p}^{M}(F)=I_{p}^{M}(\hat{F})$, where $\hat{F}$ is the symmetrization of $F$.

Now we will concentrate on multiple symmetric $\alpha$-stable integrals. The characteristic function of $M(A)$ in this case has the form

$$
E \exp \{i \theta M(A)\}=\exp \left\{-|\theta|^{\alpha} m(A)\right\}
$$

where $m$ is a $\sigma$-finite measure on $\sigma(\mathcal{S})$ that is finite on $\mathcal{S}$. Let $\psi: S \rightarrow(0, \infty)$ be such that $\int_{S} \psi(s) m(d s)=1 ; \psi$ is used to define the probability control measure $\lambda$ of $M$ as follows

$$
\lambda(A)=\int_{A} \psi(s) m(d s)
$$

Then $R(u, s)=c_{\alpha} u^{-1 / \alpha} \psi(s)^{-1 / \alpha}$, where $c_{\alpha}$ is a numerical constant (see Samorodnitsky and Taqqu (1994), p. 23). Hence

$$
M(A)=c_{\alpha} \sum_{i=1}^{\infty} \epsilon_{i} \Gamma_{i}^{-1 / \alpha} \psi\left(V_{i}\right)^{-1 / \alpha} \delta_{V_{i}}(A)
$$

Samorodnitsky and Taqqu (1990) (see also Samorodnitsky and Szulga (1989)) proved that if

$$
\left.N_{p}(f):=\int_{S^{p}}\left|f\left(s_{1}, \ldots, s_{p}\right)\right|^{\alpha}\left[1+\ln _{+} \frac{\left|f\left(s_{1}, \ldots, s_{p}\right)\right|}{\prod_{i=1}^{p} \psi\left(s_{i}\right)^{1 / \alpha}}\right)\right]^{p-1} m\left(d s_{1}\right) \cdots m\left(d s_{p}\right)<\infty
$$

then $I_{p}^{M}(f)$ exists and is by (1.5) given by

$$
I_{p}^{M}(f)=c_{\alpha}^{p} \sum_{k_{1}, \ldots, k_{p}}\left[\prod_{i=1}^{p} \epsilon_{k_{i}} \Gamma_{k_{i}}^{-1 / \alpha} \psi\left(V_{k_{i}}\right)^{-1 / \alpha}\right] f\left(V_{k_{1}}, \ldots V_{k_{p}}\right)
$$

(recall that $\log _{+} a=\log a$ if $a>1$ and $=0$ otherwise). Note that condition (1.10) depends in general on the choice of $\psi$.

This paper is organized as follows. In the next section we develop the main tool in our treatment of multiple stable integrals, the product formula. In Section 3 
we derive the asymptotic tail behavior of the distribution of a general term in the product formula, a mixed stable integral. It turns out that different terms in the product formula have different rates of decay of the tail distribution function. This fact allows us to determine the asymptotic tail behavior for the product of multiple stable integrals (Theorem 4.1) and derive from this necessary and sufficient conditions for independence of multiple stable integrals (Theorem 4.3). Finally, in Section 5 we describe briefly independence of multiple Wiener-Itô integrals, em-

phasizing differences and similarities with the stable case. The problem of finding necessary and sufficient conditions for independence of multiple integrals with respect to a general infinitely divisible random measure is still open. We conjecture that the same conditions are required here for independence as in the stable case, hence no cancellation of Poissonian jumps can occur.

\section{The product formula for multiple integrals with respect to a symmet- ric infinitely divisible random measure.}

In this section we will develop a product formula for multiple integrals with respect to symmetric infinitely divisible random measures $M$ described in Section 1. Let

$$
D^{p}=\left\{\mathbf{i} \in \mathbf{N}^{p}: \text { all } i_{1}, \ldots, i_{p} \text { are different }\right\}
$$

and

$$
D_{n}^{p}=D^{p} \cap\{1, \ldots, n\}^{p} .
$$

Lemma 2.1. Let $F: D_{n}^{p} \rightarrow \mathbf{R}, G: D_{n}^{q} \rightarrow \mathbf{R}$ be symmetric functions. Then

$$
\sum_{\mathbf{i} \in D_{n}^{p}} F(\mathbf{i}) \sum_{\mathbf{j} \in D_{n}^{q}} G(\mathbf{j})=\sum_{r=0}^{p \wedge q} r !\left(\begin{array}{l}
p \\
r
\end{array}\right)\left(\begin{array}{l}
q \\
r
\end{array}\right) \sum_{\mathbf{k} \in D_{n}^{p+q-r}} H_{r}(\mathbf{k}),
$$

where

$$
H_{r}(\mathbf{k})=F\left(k_{1}, \ldots, k_{r}, k_{r+1}, \ldots, k_{p}\right) G\left(k_{1}, \ldots, k_{r}, k_{p+1}, \ldots, k_{p+q-r}\right) .
$$


Proof. Write the left hand side of (2.3) as

$$
\sum_{r=0}^{p \wedge q} \sum_{\{(\mathbf{i}, \mathbf{j}): c(\mathbf{i}, \mathbf{j})=r\}} F(\mathbf{i}) G(\mathbf{j})
$$

where

$$
c(\mathbf{i}, \mathbf{j})=\operatorname{card}\left(\left\{i_{1}, \ldots, i_{p}\right\} \cap\left\{j_{1}, \ldots, j_{q}\right\}\right) .
$$

Consider the inner sum in (2.4). If $r=0$, then

$$
\sum_{\{(\mathbf{i}, \mathbf{j}): c(\mathbf{i}, \mathbf{j})=0\}} F(\mathbf{i}) G(\mathbf{j})=\sum_{\mathbf{k} \in D_{n}^{p+q}} H_{0}(\mathbf{k}) .
$$

If $r=c(\mathbf{i}, \mathbf{j}) \geq 1$, then $\mathbf{i}$ contains a subsequence $\mathbf{s} \in D_{n}^{r}$ such that the elements of $\mathbf{s}$ appear in certain order in $\mathbf{j}$. By the symmetry of $F, F(\mathbf{i})=F\left(\mathbf{s}, \mathbf{i}^{\prime}\right)$, where $\mathbf{i}^{\prime} \in D_{n}^{p-r}$ are the remaining elements of $\mathbf{i}$ put together after $\mathbf{s}$ was removed from $\mathbf{i}$. Similarly, $G(\mathbf{j})=G\left(\mathbf{s}, \mathbf{j}^{\prime}\right)$, and $\left(\mathbf{s}, \mathbf{i}^{\prime}, \mathbf{j}^{\prime}\right) \in D_{n}^{p+q-r}$. Hence

$$
\sum_{\{(\mathbf{i}, \mathbf{j}): c(\mathbf{i}, \mathbf{j})=r\}} F(\mathbf{i}) G(\mathbf{j})=r !\left(\begin{array}{l}
p \\
r
\end{array}\right)\left(\begin{array}{l}
q \\
r
\end{array}\right) \sum_{\mathbf{k} \in D_{n}^{p+q-r}} H_{r}(\mathbf{k}),
$$

which ends the proof.

Let $f: S^{p} \rightarrow \mathbf{R}$ and $g: S^{q} \rightarrow \mathbf{R}$ be measurable symmetric and vanishing on diagonals functions. Define

$$
\begin{aligned}
& h_{r}\left(s_{1}, \ldots, s_{p+q-r}\right) \\
& \quad=f\left(s_{1}, \ldots, s_{r}, \ldots, s_{p}\right) g\left(s_{1}, \ldots, s_{r}, s_{p+1}, \ldots, s_{p+q-r}\right) .
\end{aligned}
$$

Note that $h_{r}$ is obtained from the tensor product $f \otimes g$ by coupling the first $r$ variables of $f$ and $g$. As a function of the first $\mathrm{r}$ variables only $h_{r}$ is symmetric and vanishes on diagonals, but in general, $h_{r}$ is neither symmetric nor vanishes on the diagonals. Let $M$ be a random measure given by (1.4). Since $\lambda$ is atomless, the quadratic variation $[M]$ of $M$ is given by

$$
[M](A)=\sum_{i=1}^{\infty} \xi_{i}^{2} \delta_{V_{i}}(A), \quad A \in \mathcal{S} .
$$

Let $I_{r}^{[M]}(\cdot)$ denote $r$-tuple integral with respect to $[M]$. 
Lemma 2.2. Suppose $I_{p}^{M}(f)$ and $I_{q}^{M}(g)$ exist (see (1.5)-(1.6)). Then, for every $1 \leq r \leq p \wedge q$ and for $\lambda^{\otimes(p+q-2 r)}$-almost all $\left(s_{r+1}, \ldots, s_{p+q-r}\right) \in S^{p+q-2 r}$, the series

$$
H_{r}\left(\cdot, s_{r+1}, \ldots, s_{p+q-r}\right)=\sum_{\mathbf{k} \in D^{r}} \xi_{k_{1}}^{2} \ldots \xi_{k_{r}}^{2} h_{r}\left(V_{k_{1}}, \ldots, V_{k_{r}}, s_{r+1}, \ldots, s_{p+q-r}\right)
$$

converges absolutely with probability one. Hence

$$
I_{r}^{[M]}\left(h_{r}\left(\cdot, s_{r+1}, \ldots, s_{p+q-r}\right)\right)=H_{r}\left(\cdot, s_{r+1}, \ldots, s_{p+q-r}\right) .
$$

Proof. Applying Th. 4.7 in Kallenberg and Szulga (1989) to the marked Poisson point process $\left\{\left(\left|\xi_{i}\right|, V_{i}\right)\right\}_{i \in \mathbf{N}}$ we get that for $\lambda^{\otimes(p-r)}$-almost all $\left(s_{r+1}, \ldots, s_{p}\right) \in S^{p-r}$ the iterated integral

$$
I_{r}^{M}\left(f\left(\cdot, s_{r+1}, \ldots, s_{p}\right)\right)
$$

exists and $I_{p-r}^{M}\left(I_{r}^{M}(f)\right)=I_{p}^{M}(f)$. The analogous statement holds also for $g$. Hence

$$
\begin{aligned}
\sum_{\mathbf{k} \in D^{r}} \xi_{k_{1}}^{2} \cdots \xi_{k_{r}}^{2} & \left|h_{r}\left(V_{k_{1}}, \ldots, V_{k_{r}}, s_{r+1}, \ldots, s_{p+q-r}\right)\right| \\
\leq & \left(\sum_{\mathbf{k} \in D^{r}} \xi_{k_{1}}^{2} \cdots \xi_{k_{r}}^{2} f^{2}\left(V_{k_{1}}, \ldots, V_{k_{r}}, s_{r+1}, \ldots, s_{p}\right)\right)^{1 / 2} \\
& \times\left(\sum_{\mathbf{k} \in D^{r}} \xi_{k_{1}}^{2} \cdots \xi_{k_{r}}^{2} g^{2}\left(V_{k_{1}}, \ldots, V_{k_{r}}, s_{p+1}, \ldots, s_{p+q-r}\right)\right)^{1 / 2}<\infty \text { a.s. }
\end{aligned}
$$

The finiteness of the last two series comes from (1.6) applied to the iterated integrals $I_{r}^{M}\left(f\left(\cdot, s_{r+1}, \ldots, s_{p}\right)\right)$ and $I_{r}^{M}\left(g\left(\cdot, s_{p+1}, \ldots, s_{p+q-r}\right)\right)$.

Theorem 2.3. Suppose $I_{p}^{M}(f)$ and $I_{q}^{M}(g)$ exist. Then

$$
I_{p}^{M}(f) I_{q}^{M}(g)=\sum_{r=0}^{p \wedge q} r !\left(\begin{array}{l}
p \\
r
\end{array}\right)\left(\begin{array}{l}
q \\
r
\end{array}\right)\left(I_{p+q-2 r}^{M} \otimes I_{r}^{[M]}\right)\left(h_{r}\right) .
$$

Here $\left(I_{p+q-2 r}^{M} \otimes I_{r}^{[M]}\right)\left(h_{r}\right):=I_{p+q-2 r}^{M}\left(J_{r}\right)$, where $J_{r}$ is given by (2.7).

Proof. The existence of $I_{p+q-2 r}^{M}\left(J_{r}\right)$ follows from (1.8); one needs to apply (2.8) and the conditions on the existence of $I_{p}^{M}(f)$ and of $I_{q}^{M}(g)$. The rest of the proof is a direct application of Lemma 2.1. 
Next we will examine the product of symmetric multiple stable integrals. From (1.9) the quadratic variation $[M]$ of $M$ is a positive $\alpha$-stable random measure with the series representation

$$
[M](A)=c_{\alpha}^{2} \sum_{i=1}^{\infty} \Gamma_{i}^{-2 / \alpha} \psi\left(V_{i}\right)^{-2 / \alpha} \delta_{V_{i}}(A) .
$$

Hence the product $I_{p}^{M}(f) I_{q}^{M}(g)$ is the sum of the $\alpha$-stable multiple integral $I_{p+q}^{M}\left(h_{0}\right)$ and mixed, $\alpha$ and $\alpha / 2$-stable, multiple integrals $\left(I_{p+q-2 r}^{M} \otimes I_{r}^{[M]}\right)\left(h_{r}\right)$, where

$$
\begin{aligned}
\left(I_{p+q-2 r}^{M} \otimes I_{r}^{[M]}\right)\left(h_{r}\right)= & c_{\alpha}^{p+q} \sum_{\mathbf{k} \in D^{p+q-r}}\left[\prod_{i=1}^{r} \Gamma_{k_{i}}^{-2 / \alpha} \psi\left(V_{k_{i}}\right)^{-2 / \alpha}\right] \\
& {\left[\prod_{j=1}^{p+q-2 r} \epsilon_{k_{r+j}} \Gamma_{k_{r+j}}^{-1 / \alpha} \psi\left(V_{k_{r+j}}\right)^{-1 / \alpha}\right] h_{r}\left(V_{k_{1}}, \ldots V_{k_{p+q-r}}\right) . }
\end{aligned}
$$

We will deduce the conditions for independence of $I_{p}^{M}(f)$ and $I_{q}^{M}(g)$ by computing the tail behavior of the product $I_{p}^{M}(f) I_{q}^{M}(g)$. We will show that the tails of $(2.10)$ have different rates of decay for different $r$ 's and exactly one of them determines the tail behavior of the product (2.9).

\section{Tail behavior of the distribution of mixed stable integrals.}

The product formula established in the previous section shows that for two symmetric stable integrals $I_{p}^{M}(f)$ and $I_{q}^{M}(g)$ the product $I_{p}^{M}(f) I_{q}^{M}(g)$ is a linear combination of a $p+q$-tuple symmetric stable integral $I_{p+q}^{M}\left(h_{0}\right)$ and mixed stable integrals. The tail behavior of the distribution of $I_{p+q}^{M}\left(h_{0}\right)$ is known:

$$
\lim _{\lambda \rightarrow \infty} \lambda^{\alpha}(\log \lambda)^{-(p+q)+1} P\left(\left|I_{p+q}^{M}\left(h_{0}\right)\right|>\lambda\right)=k_{0, \alpha}\left\|\widehat{h_{0}}\right\|_{L^{\alpha}\left(S^{p+q}\right)}^{\alpha}
$$

where $\widehat{h_{0}}$ is the symmetrization of $h_{0}$ and $k_{0, \alpha}$ is positive constant, see Samorodnitsky and Szulga (1989) and Samorodnitsky and Taqqu (1990). To establish the tail behavior of the distribution of the product $I_{p}^{M}(f) I_{q}^{M}(g)$ we need to understand the tail behavior of the distribution of mixed stable integrals, and this is our goal 
in this section. Let, therefore, $p$ and $q$ be two positive integers, and let $r \leq p \wedge q$ be another positive integer. We consider the mixed stable integral $\left(I_{p+q-2 r}^{M} \otimes I_{r}^{[M]}\right)\left(h_{r}\right)$ with the kernel $h_{r}$ given by (2.5). The following is the main result of this section.

Theorem 3.1. Suppose that $N_{p}(f)<\infty$ and $N_{q}(g)<\infty($ see (1.10)). Then

$$
\lim _{\lambda \rightarrow \infty} \lambda^{\alpha / 2}(\log \lambda)^{-r+1} P\left(\left|\left(I_{p+q-2 r}^{M} \otimes I_{r}^{[M]}\right)\left(h_{r}\right)\right|>\lambda\right)=k_{r, \alpha} C_{r}(f, g),
$$

where $k_{r, \alpha}=c_{\alpha}^{r \alpha}(r !)^{\alpha / 2-1} /(r-1) !$ and

$$
C_{r}(f, g)=\int_{S^{r}} E\left|I_{p+q-2 r}\left(\hat{h}_{r}\left(s_{1}, \ldots, s_{r}, \cdot\right)\right)\right|^{\alpha / 2} m\left(d s_{1}\right) \ldots m\left(d s_{r}\right)<\infty .
$$

Here $\hat{h}_{r}$ is the symmetrization of $h_{r}$ with respect to variables $s_{r+1}, \ldots, s_{p+q-r}$, i.e.,

$$
\hat{h}_{r}\left(s_{1}, \ldots, s_{p+q-r}\right)=((p+q-2 r) !)^{-1} \sum_{\pi} h_{r}\left(s_{1}, \ldots, s_{r}, s_{\pi(r+1)}, \ldots, s_{\pi(p+q-r)}\right)
$$

where $\pi$ runs over all permutations of the numbers $\{r+1, \ldots, p+q-r\}$. Finally, $I_{p+q-2 r}\left(\hat{h}_{r}\left(s_{1}, \ldots, s_{r}, \cdot\right)\right)$ is the ordinary (i.e. not mixed) multiple integral of order $p+q-2 r$ with respect to the symmetric $\alpha$-stable random measure $M$ of $\hat{h}_{r}$ taken as a function of its last $p+q-2 r$ coordinates.

Proof. We use the series representation of $\left(I_{p+q-2 r}^{M} \otimes I_{r}^{[M]}\right)\left(h_{r}\right)$ given by $(2.10)$. Recall that $V_{1}, V_{2}, \ldots$ are i.i.d. with common distribution $\psi(s) m(d s), \epsilon_{1}, \epsilon_{2}, \ldots$ are i.i.d. Rademacher random variables and $\Gamma_{1}, \Gamma_{2}, \ldots$ are the ordered points of a standard Poisson process on $(0, \infty)$, with all three sequences being independent. Let

$$
\begin{aligned}
M_{r}:=r !\left[\prod_{i=1}^{r} \Gamma_{i}^{-2 / \alpha} \psi\left(V_{i}\right)^{-2 / \alpha}\right] \sum_{\mathbf{j} \in D^{p+q-2 r}}\left[\prod_{k=1}^{p+q-2 r} \epsilon_{j_{k}+r} \Gamma_{j_{k}+r}^{-1 / \alpha} \psi\left(V_{j_{k}+r}\right)^{-1 / \alpha}\right] \\
h_{r}\left(V_{1}, \ldots, V_{r}, V_{j_{1}+r}, \ldots, V_{j_{p+q}-2 r}+r\right) .
\end{aligned}
$$

We think of $M_{r}$ as the "main term" of $c_{\alpha}^{-p-q}\left(I_{p+q-2 r}^{M} \otimes I_{r}^{[M]}\right)\left(h_{r}\right)$. The proof is divided into two main steps. The first one shows that

$$
\lim _{\lambda \rightarrow \infty} \lambda^{\alpha / 2}(\log \lambda)^{-r+1} P\left(\left|M_{r}\right|>\lambda\right)=\frac{c_{\alpha}^{-(p+q-2 r) \alpha / 2}(r !)^{\alpha / 2-1}}{(r-1) !} C_{r}(f, g)
$$


In the second step we show that the tail of the remainder

$$
R_{r}:=c_{\alpha}^{-p-q}\left(I_{p+q-2 r}^{M} \otimes I_{r}^{[M]}\right)\left(h_{r}\right)-M_{r}
$$

is of the smaller order than $M_{r}$. Specifically, we will prove that

$$
\lim _{\lambda \rightarrow \infty} \lambda^{\alpha / 2}(\log \lambda)^{-r+1} P\left(\left|R_{r}\right|>\lambda\right)=0
$$

Clearly, (3.4)-(3.6) give (3.1) and so conclude the proof. Despite a clear structure of this proof, the actual computations leading to (3.4) and (3.6) are intricate and lengthly; particularly (3.6) involves several truncation arguments and decoupling.

We begin by proving (3.4). Recall that, conditional on $\Gamma_{r+1}$, the joint distribution of $\Gamma_{1} / \Gamma_{r+1}, \ldots, \Gamma_{r} / \Gamma_{r+1}$ is that of order statistics from i.i.d. uniform sample of size $r$ in $(0,1)$. Hence

$$
P\left(\frac{1}{r !}\left|M_{r}\right|>\lambda\right)=\int_{0}^{\infty} e^{-x} \frac{x^{r}}{r !} P_{x}(\lambda) d x
$$

where

$$
\begin{aligned}
P_{x}(\lambda): & =P\left(\left[\prod_{i=1}^{r}\left(x U_{i}\right)^{-2 / \alpha} \psi\left(V_{i}\right)^{-2 / \alpha}\right]\right. \\
& \mid \sum_{\mathbf{j} \in D^{p+q-2 r}}\left[\prod_{k=1}^{p+q-2 r} \epsilon_{j_{k}+r}\left(x+\Gamma_{j_{k}-1}\right)^{-1 / \alpha} \psi\left(V_{j_{k}+r}\right)^{-1 / \alpha}\right] \\
& \left.h_{r}\left(V_{1}, \ldots, V_{r}, V_{j_{1}+r}, \ldots, V_{j_{p+q-2 r}+r}\right) \mid>\lambda\right) .
\end{aligned}
$$

The sequences $\epsilon_{1}, \epsilon_{2}, \ldots, V_{1}, V_{2}, \ldots$ and $\Gamma_{1}, \Gamma_{2}, \ldots$ in (3.8) are as before, and $U_{1}, \ldots, U_{r}$ are independent of them i.i.d. uniform in $(0,1)$ random variables. Further, $\Gamma_{0}=0$.

To establish the asymptotic behavior of $P_{x}(\lambda)$ we need the following lemma.

Lemma 3.2. Let $Y$ be a nonnegative random variable with $E Y<\infty$ independent of i.i.d. uniform in $(0,1)$ random variables $U_{1}, \ldots, U_{r}, r \geq 1$. Then

$$
\lim _{\lambda \rightarrow \infty} \lambda(\log \lambda)^{-(r-1)} P\left(\prod_{j=1}^{r} U_{j}^{-1} Y>\lambda\right)=\frac{1}{(r-1) !} E Y .
$$


Moreover, there is a finite positive constant $K=K(r)$ depending only on $r$ such that for all $\lambda>0$,

$$
P\left(\prod_{j=1}^{r} U_{j}^{-1} Y>\lambda\right) \leq K \lambda^{-1}\left(1+\log _{+} \lambda\right)^{r-1}(1+E Y) .
$$

Proof of Lemma 3.2. Let $F_{Y}$ be the law of $Y$. We have

$$
P\left(\prod_{j=1}^{r} U_{j}^{-1} Y>\lambda\right)=P(Y>\lambda)+\int_{0}^{\lambda} P\left(\prod_{j=1}^{r} U_{j}^{-1}>\frac{\lambda}{y}\right) F_{Y}(d y) .
$$

The assumption $E Y<\infty$ implies that

$$
P(Y>\lambda) \leq \lambda^{-1} E Y
$$

$\lambda>0$. Furthermore,

$$
\begin{aligned}
\int_{0}^{\lambda} P\left(\prod_{j=1}^{r} U_{j}^{-1}>\frac{\lambda}{y}\right) F_{Y}(d y) & =\int_{0}^{\lambda} P\left(\Gamma_{r}>\log \frac{\lambda}{y}\right) F_{Y}(d y) \\
& =\lambda^{-1} \sum_{i=0}^{r-1} \frac{1}{i !} \int_{0}^{\lambda} y\left(\log \frac{\lambda}{y}\right)^{i} F_{Y}(d y) .
\end{aligned}
$$

Next we observe that for every $i \geq 0$

$$
\begin{aligned}
\int_{0}^{\lambda} y\left(1+\log \frac{\lambda}{y}\right)^{i} F_{Y}(d y) & \leq \sup _{0<y \leq \lambda^{-1 / 2}} y\left(1+\log \frac{\lambda}{y}\right)^{i}+\int_{\lambda^{-1 / 2}}^{\lambda} y\left(1+\log \frac{\lambda}{y}\right)^{i} F_{Y}(d y) \\
& \leq \lambda \sup _{x \geq \lambda^{3 / 2}} \frac{(1+\log x)^{i}}{x}+\left(1+\frac{3}{2} \log \lambda\right)^{i} \int_{\lambda^{-1 / 2}}^{\lambda} y F_{Y}(d y) \\
& \leq c \lambda^{-1 / 2}(\log \lambda)^{i}+c E Y(\log \lambda)^{i}
\end{aligned}
$$

for all $\lambda>3$ (say). Here $c$ is a positive constant that depends only on $i$. Now the bound (3.10) follows immediately from (3.12), (3.13) and (3.14). Furthermore, one can see by (3.11) and (3.13) that to derive (3.9) one has only to establish that

$$
\lim _{\lambda \rightarrow \infty}(\log \lambda)^{-j} \int_{0}^{\lambda} y\left(\log \frac{\lambda}{y}\right)^{j} F_{Y}(d y)=E Y
$$


However, (3.15) follows upon observing that by the argument in (3.14)

$$
\lim _{\lambda \rightarrow \infty}(\log \lambda)^{-j} \int_{0}^{\lambda} y\left(\log \frac{\lambda}{y}\right)^{j} F_{Y}(d y)=\lim _{\lambda \rightarrow \infty}(\log \lambda)^{-j} \int_{\lambda^{-1 / 2}}^{\lambda} y\left(\log \frac{\lambda}{y}\right)^{j} F_{Y}(d y)
$$

and using a dominated convergence argument.

Let

$$
\begin{gathered}
Y_{x}:=\prod_{i=1}^{r} \psi\left(V_{i}\right)^{-1} \mid \sum_{\mathbf{j} \in D^{p+q-2 r}}\left[\prod_{k=1}^{p+q-2 r} \epsilon_{j_{k}+r}\left(x+\Gamma_{j_{k}-1}\right)^{-1 / \alpha} \psi\left(V_{j_{k}+r}\right)^{-1 / \alpha}\right] \\
\left.h_{r}\left(V_{1}, \ldots, V_{r}, V_{j_{1}+r}, \ldots, V_{j_{p+q-2 r}+r}\right)\right|^{\alpha / 2}
\end{gathered}
$$

where $x>0$ is arbitrary but fixed. We have

$$
P_{x}(\lambda)=P\left(\prod_{i=1}^{r} U_{i}^{-1} Y_{x}>\lambda^{\alpha / 2} x^{r}\right) .
$$

We will show how the first step of the proof can be completed when we have

$$
\begin{aligned}
E\left[\prod_{i=1}^{r} \psi\left(V_{i}\right)^{-1} \mid \sum_{\mathbf{j} \in D^{p+q-2 r}}\left[\prod_{k=1}^{p+q-2 r} \epsilon_{j_{k}+r} \Gamma_{j_{k}}^{-1 / \alpha} \psi\left(V_{j_{k}+r}\right)^{-1 / \alpha}\right]\right. \\
\left.\left.h_{r}\left(V_{1}, \ldots, V_{r}, V_{j_{1}+r}, \ldots, V_{j_{p+q-2 r}+r}\right)\right|^{\alpha / 2}\right]<\infty
\end{aligned}
$$

and

$$
\begin{aligned}
& E\left[\left(\prod_{i=1}^{r} \psi\left(V_{i}\right)^{-1}\right) \psi\left(V_{r+1}\right)^{-1 / 2} \mid \sum_{\mathbf{j} \in D^{p+q-2 r-1}}\left[\prod_{k=1}^{p+q-2 r-1} \epsilon_{j_{k}+r+1} \Gamma_{j_{k}}^{-1 / \alpha} \psi\left(V_{j_{k}+r+1}\right)^{-1 / \alpha}\right]\right. \\
& \left.\left.(3.18) \quad h_{r}\left(V_{1}, \ldots, V_{r}, V_{r+1}, V_{j_{1}+r+1}, \ldots, V_{j_{p+q-2 r-1}+r+1}\right)\right|^{\alpha / 2}\right]<\infty .
\end{aligned}
$$

(of course, (3.18) is meaningful only if $2 r<p+q$ ). First, using Khinchine's inequality for tetrahedral sums in Rademacher random variables we get that there is a finite $M>0$ such that for every $x>0$

$$
\begin{array}{r}
E\left[\prod_{i=1}^{r} \psi\left(V_{i}\right)^{-1} \mid \sum_{\mathbf{j} \in D^{p+q-2 r}}\left[\prod_{k=1}^{p+q-2 r} \epsilon_{j_{k}+r}\left(x+\Gamma_{j_{k}}\right)^{-1 / \alpha} \psi\left(V_{j_{k}+r}\right)^{-1 / \alpha}\right]\right. \\
\left.\left.h_{r}\left(V_{1}, \ldots, V_{r}, V_{j_{1}+r}, \ldots, V_{j_{p+q-2 r}+r}\right)\right|^{\alpha / 2}\right] \leq M
\end{array}
$$


and

$$
\begin{aligned}
& E\left[\left(\prod_{i=1}^{r} \psi\left(V_{i}\right)^{-1}\right) \psi\left(V_{r+1}\right)^{-1 / 2}\right. \\
& \quad \mid \sum_{\mathbf{j} \in D^{p+q-2 r-1}}\left[\prod_{k=1}^{p+q-2 r-1} \epsilon_{j_{k}+r+1}\left(x+\Gamma_{j_{k}}\right)^{-1 / \alpha} \psi\left(V_{j_{k}+r+1}\right)^{-1 / \alpha}\right] \\
& \left.\left.\quad h_{r}\left(V_{1}, \ldots, V_{r}, V_{r+1}, V_{j_{1}+r+1}, \ldots, V_{j_{p+q-2 r-1}+r+1}\right)\right|^{\alpha / 2}\right] \leq M .
\end{aligned}
$$

Splitting the inner sum in $Y_{x}$ accordingly to $j_{k} \geq 2$ for all $k$ and $j_{k}=1$ for some $k$ we get

$$
Y_{x} \leq Y_{x}^{\prime}+r x^{-1 / 2} Y_{x}^{\prime \prime}
$$

where $Y_{x}^{\prime}$ and $Y_{x}^{\prime \prime}$ are the integrands in (3.19) and (3.20), respectively. Hence $E Y_{x}<\infty$ and, by (3.16) and Lemma 3.2,

$$
\lim _{\lambda \rightarrow \infty} \lambda^{\alpha / 2}(\log \lambda)^{-(r-1)} P_{x}(\lambda)=\frac{x^{-r}}{(r-1) !} E Y_{x}
$$

Hence, making a formal passage to the limit in (3.7),

$$
\begin{aligned}
& \lim _{\lambda \rightarrow \infty} \lambda^{\alpha / 2}(\log \lambda)^{-(r-1)} P\left(\left|M_{r}\right|>\lambda\right) \\
& =\lim _{\lambda \rightarrow \infty} \lambda^{\alpha / 2}(\log \lambda)^{-(r-1)} \int_{0}^{\infty} e^{-x} \frac{x^{r}}{r !} P_{x}\left(\frac{\lambda}{r !}\right) d x \\
& =\int_{0}^{\infty} e^{-x} \frac{x^{r}}{r !}(r !)^{\alpha / 2} \frac{x^{-r}}{(r-1) !} E Y_{x} d x \\
& =\frac{(r !)^{\alpha / 2-1}}{(r-1) !} E\left[\prod_{i=1}^{r} \psi\left(V_{i}\right)^{-1} \mid \sum_{\mathbf{j} \in D^{p+q-2 r}}\left[\prod_{k=1}^{p+q-2 r} \epsilon_{j_{k}} \Gamma_{j_{k}}^{-1 / \alpha} \psi\left(V_{j_{k}+r}\right)^{-1 / \alpha}\right]\right. \\
& =\frac{c_{\alpha}^{-(p+q-2 r) \alpha / 2}(r !)^{\alpha / 2-1}}{(r-1) !} \int_{S^{r}} E\left|I_{p+q-2 r}\left(\hat{h}_{r}\left(s_{1}, \ldots, s_{r}, \cdot\right)\right)\right|^{\alpha / 2} m\left(d s_{1}\right) \ldots m\left(d s_{r}\right),
\end{aligned}
$$

which is exactly (3.4). To complete this first step of the proof we need to justify the interchange of the limit and integral in the above derivation. Using (3.10) of 
Lemma 3.2 with (3.19) and (3.20) we get

$$
\begin{aligned}
P_{x}(\lambda) & \leq P\left(\left(\prod_{i=1}^{r} U_{i}^{-1}\right)\left(Y_{x}^{\prime}+r x^{-1 / 2} Y_{x}^{\prime \prime}\right)>\lambda^{\alpha / 2} x^{r}\right) \\
& \leq P\left(\left(\prod_{i=1}^{r} U_{i}^{-1}\right) Y_{x}^{\prime}>\frac{1}{2} \lambda^{\alpha / 2} x^{r}\right)+P\left(\left(\prod_{i=1}^{r} U_{i}^{-1}\right) Y_{x}^{\prime \prime}>\frac{1}{2 r} \lambda^{\alpha / 2} x^{r+1 / 2}\right) \\
& \leq c \lambda^{-\alpha / 2}\left(1+\log _{+} \lambda\right)^{r-1}\left(x^{-r} \vee x^{-(r+1 / 2)}\right)\left(1+\log _{+} x\right)^{r-1}
\end{aligned}
$$

for all $\lambda>0$ and $x>0$, where $c$ is a finite positive constant. Therefore, the interchange the limit and the integral is legitimate by the dominated convergence theorem. This proves (3.4) modulo proving (3.17) and (3.18), which we do presently. Since the required argument is similar in both cases, we give the details only for (3.17).

We start with checking the following statement. For every $J=0,1, \ldots, p+q-2 r$,

$$
E\left[\left(\prod_{i=1}^{r} \psi\left(V_{i}\right)^{-1} \prod_{d=r+1}^{r+J} \psi\left(V_{d}\right)^{-1 / 2} \Gamma_{d-r}^{-1 / 2}\right) \mid \sum_{\mathbf{j} \in D^{p+q-2 r-J}}\right.
$$

$$
\left.\left.\left[\prod_{k=1}^{p+q-2 r-J} \epsilon_{j_{k}+r+J} \Gamma_{j_{k}+J}^{-1 / \alpha} \psi\left(V_{j_{k}+r+J}\right)^{-1 / \alpha}\right] h_{r}\left(V_{1}, \ldots, V_{r}, \mathbf{W}_{\mathbf{j}}\right)\right|^{\alpha / 2}\right]<\infty
$$

where for every $\mathbf{j} \in D^{p+q-2 r-J}, \mathbf{W}_{\mathbf{j}}$ is a $(p+q-2 r)$-dimensional random vector in which $J$ coordinates are $V_{r+1}, \ldots, V_{r+J}$ and the remaining $p+q-2 r-J$ coordinates are $V_{j_{1}+r+J}, \ldots, V_{j_{p+q-2 r-J}+r+J}$.

If $J=p+q-2 r$ then (3.21) is trivially true, so we will prove (3.21) by downward induction in $J$. Assume that (3.21) holds for all $J>J_{0}$, for some $J_{0}$ such that $1 \leq J_{0}+1 \leq p+q-2 r$, and let us prove it for $J=J_{0}$. Note that the assumption of the induction and the Khinchine inequality for tetrahedral sums in Rademacher random variables implies that the finiteness of the expectation in (3.21) for $J>J_{0}$ will be preserved if we restrict the sum to a nonrandom subset of $D^{p+q-2 r-J}$. The first step in the inductive procedure is to note that it is enough to prove that for 
some $m \geq 1$

$$
E\left[\left(\prod_{i=1}^{r} \psi\left(V_{i}\right)^{-1} \prod_{d=r+1}^{r+J_{0}} \psi\left(V_{d}\right)^{-1 / 2} \Gamma_{d-r}^{-1 / 2}\right) \mid \sum_{\substack{\mathbf{j} \in D^{p+q-2 r-J_{0}} \\ j_{k} \geq m, k \leq p+q-2 r-J_{0}}}\right.
$$

$$
\left.\left.\left[\prod_{k=1}^{p+q-2 r-J_{0}} \epsilon_{j_{k}+r+J_{0}} \Gamma_{j_{k}+J_{0}}^{-1 / \alpha} \psi\left(V_{j_{k}+r+J_{0}}\right)^{-1 / \alpha}\right] h_{r}\left(V_{1}, \ldots, V_{r}, \mathbf{W}_{\mathbf{j}}\right)\right|^{\alpha / 2}\right]<\infty
$$

because the absolute difference between the random variables in the left hand sides of (3.21) and (3.22) is bounded above by the sum of a finite number of random variables each one of which is of the type given in the left hand side of (3.21) with $J>J_{0}$ and the sum taken over a nonrandom subset of $D^{p+q-2 r-J}$. For such random variables we use the assumption of the induction to establish finiteness of the corresponding moment. Let us, therefore, show that for some $m \geq 1$ (3.22) holds.

Using once again the Khinchine inequality for tetrahedral sums in Rademacher random variables we see that for some positive constant $c$ (that is allowed to change from one place to another) the expectation in the left hand side of (3.22) is bounded above by

$$
\begin{aligned}
& c E\left[\left(\prod_{i=1}^{r} \psi\left(V_{i}\right)^{-1} \prod_{d=r+1}^{r+J_{0}} \psi\left(V_{d}\right)^{-1 / 2} \Gamma_{d-r}^{-1 / 2}\right) \mid \sum_{\substack{\mathbf{j} \in D^{p+q-2 r-J_{0}} \\
j_{k} \geq m, k \leq p+q-2 r-J_{0}}}\right. \\
& \left.\left.\left[\prod_{k=1}^{p+q-2 r-J_{0}} \Gamma_{j_{k}+J_{0}}^{-2 / \alpha} \psi\left(V_{j_{k}+r+J_{0}}\right)^{-2 / \alpha}\right]\left|h_{r}\left(V_{1}, \ldots, V_{r}, V_{r+1}, \mathbf{W}_{\mathbf{j}}\right)\right|^{2}\right|^{\alpha / 4}\right] \\
& \leq c E\left[\left(\prod_{i=1}^{r} \psi\left(V_{i}\right)^{-1} \prod_{d=r+1}^{r+J_{0}} \psi\left(V_{d}\right)^{-1 / 2}\right) \mid \sum_{\substack{\mathbf{j} \in D^{p+q-2 r-J_{0}} \\
j_{k} \geq m, k \leq p+q-2 r-J_{0}}}\right. \\
& \left.\left.\left[\prod_{k=1}^{p+q-2 r-J_{0}} \Gamma_{j_{k}}^{-2 / \alpha} \psi\left(V_{j_{k}+r+J_{0}}\right)^{-2 / \alpha}\right]\left|h_{r}\left(V_{1}, \ldots, V_{r}, V_{r+1}, \mathbf{W}_{\mathbf{j}}\right)\right|^{2}\right|^{\alpha / 4}\right] \\
& \leq c \sum_{K=0 \vee J_{0}-q-r}^{J_{0} \wedge(p-r)} E\left[\prod _ { i = 1 } ^ { r } \psi ( V _ { i } ) ^ { - 1 } \left(\prod_{d=r+1}^{r+K} \psi\left(V_{d}\right)^{-2 / \alpha} \sum_{j_{1}=m}^{\infty} \ldots \sum_{j_{p-r}-K=m}^{\infty}\right.\right.
\end{aligned}
$$




$$
\begin{aligned}
& {\left[\prod_{k=1}^{p-r-K} \Gamma_{j_{k}}^{-2 / \alpha} \psi\left(V_{j_{k}+r+J_{0}}\right)^{-2 / \alpha}\right] \mid f\left(V_{1}, \ldots, V_{r}, V_{r+1}, \ldots, V_{r+K}, V_{j_{1}+r+J_{0}}\right.} \\
& \left.\left.\ldots, V_{j_{p-r-K}+r+J_{0}}\right)\left.\right|^{2}\right)^{\alpha / 4}\left(\prod_{d=r+K+1}^{r+J_{0}} \psi\left(V_{d}\right)^{-2 / \alpha} \sum_{j_{1}=m}^{\infty} \ldots \sum_{j_{q-r-J_{0}+K}=m}^{\infty}\right. \\
& {\left[\prod_{k=1}^{q-r-J_{0}+K} \Gamma_{j_{k}}^{-2 / \alpha} \psi\left(V_{j_{k}+r+J_{0}}\right)^{-2 / \alpha}\right] \mid g\left(V_{1}, \ldots, V_{r}, V_{r+K+1}, \ldots, V_{r+J_{0}}, V_{j_{1}+r+J_{0}},\right.} \\
& \left.\left.\left.\ldots, V_{j_{q-r-J_{0}+K}+r+J_{0}}\right)\left.\right|^{2}\right)^{\alpha / 4}\right] \\
& \leq c \sum_{K=0 \vee J_{0}-q-r}^{J_{0} \wedge(p-r)}\left[E \left(\prod_{d=1}^{r+K} \psi\left(V_{d}\right)^{-2 / \alpha}\right.\right. \\
& \sum_{j_{1}=m}^{\infty} \ldots \sum_{j_{p}-r-K=m}^{\infty}\left[\prod_{k=1}^{p-r-K} \Gamma_{j_{k}}^{-2 / \alpha} \psi\left(V_{j_{k}+r+J_{0}}\right)^{-2 / \alpha}\right] \\
& \left.\left.\left|f\left(V_{1}, \ldots, V_{r}, V_{r+1}, \ldots, V_{r+K}, V_{j_{1}+r+J_{0}}, \ldots, V_{j_{p-r-K}+r+J_{0}}\right)\right|^{2}\right)^{\alpha / 2}\right]^{1 / 2} \\
& {\left[E \left(\prod_{d=1}^{r+J_{0}-K} \psi\left(V_{d}\right)^{-2 / \alpha} \sum_{j_{1}=m}^{\infty} \ldots \sum_{j_{q-r}-J_{0}+K=m}^{\infty}\left[\prod_{k=1}^{q-r-J_{0}+K} \Gamma_{j_{k}}^{-2 / \alpha} \psi\left(V_{j_{k}+r+J_{0}}\right)^{-2 / \alpha}\right]\right.\right.} \\
& \left.\left.\left|g\left(V_{1}, \ldots, V_{r}, V_{r+1}, \ldots, V_{r+J_{0}-K}, V_{j_{1}+r+J_{0}}, \ldots, V_{j_{q-r-J_{0}+K}+r+J_{0}}\right)\right|^{2}\right)^{\alpha / 2}\right]^{1 / 2} \\
& :=c \sum_{K=0 \vee J_{0}-q-r}^{J_{0} \wedge(p-r)}\left(E S_{1, K}^{\alpha / 2}\right)^{1 / 2}\left(E S_{2, K}^{\alpha / 2}\right)^{1 / 2}
\end{aligned}
$$

Hence, to establish (3.22) it is enough to prove that for some $m \geq 1 E S_{1, K}^{\alpha / 2}<\infty$ and $E S_{2, K}^{\alpha / 2}<\infty$. Since the two statement are of the same kind, we only check that

$$
E S_{1, K}^{\alpha / 2}<\infty
$$

For a $\mathbf{j}=\left(j_{1}, \ldots, j_{p-r-K}\right)$ with $j_{k} \geq m, 1 \leq k \leq p-r-K$ we define

$$
\begin{aligned}
X_{\mathbf{j}} & =\prod_{d=1}^{r+K} \psi\left(V_{d}\right)^{-2 / \alpha} \prod_{k=1}^{p-r-K} \psi\left(V_{j_{k}+r+J_{0}}\right)^{-2 / \alpha} \\
& \left|f\left(V_{1}, \ldots, V_{r}, V_{r+1}, \ldots, V_{r+K}, V_{j_{1}+r+J_{0}}, \ldots, V_{j_{p-r-K}+r+J_{0}}\right)\right|^{2} .
\end{aligned}
$$


Then the distribution of $X_{\mathbf{j}}$ does not depend on $\mathbf{j}$, and

$$
\begin{aligned}
E S_{1, K}^{\alpha / 2} & =E\left(\sum_{j_{1}=m}^{\infty} \ldots \sum_{j_{p-r-K}=m}^{\infty}\left(\prod_{k=1}^{p-r-K} \Gamma_{j_{k}}^{-2 / \alpha}\right) X_{\mathbf{j}}\right)^{\alpha / 2} \\
& \leq E\left(\sum_{j_{1}=m}^{\infty} \ldots \sum_{j_{p-r-K}=m}^{\infty}\left(\prod_{k=1}^{p-r-K} \Gamma_{j_{k}}^{-2 / \alpha}\right) X_{\mathbf{j}} \mathbf{1}\left(X_{\mathbf{j}}^{\alpha / 2} \leq[\mathbf{j}]\right)\right)^{\alpha / 2} \\
& +E\left(\sum_{j_{1}=m}^{\infty} \ldots \sum_{j_{p-r-K}=m}^{\infty}\left(\prod_{k=1}^{p-r-K} \Gamma_{j_{k}}^{-2 / \alpha}\right) X_{\mathbf{j}} \mathbf{1}\left(X_{\mathbf{j}}^{\alpha / 2}>[\mathbf{j}]\right)\right)^{\alpha / 2} \\
& :=E S_{11}^{\alpha / 2}+E S_{12}^{\alpha / 2} .
\end{aligned}
$$

Here $[\mathbf{j}]=\prod_{k=1}^{p-r-K} j_{k}$. Choose now

$$
m>\frac{2}{\alpha}(p \vee q)
$$

We now use Proposition 5.1 of Samorodnitsky and Szulga (1989) to conclude that

$$
E S_{11}^{\alpha / 2} \leq c\left(E\left(X^{\alpha / 2}\left(1+\log _{+} X\right)^{p-r-K-1}\right)\right)^{\alpha / 2}
$$

Here $X$ is a generic random variable with the same law as $X_{\mathbf{j}}$, any $\mathbf{j}$. Now (3.1) immediately gives us

$$
\begin{aligned}
E S_{11}^{\alpha / 2} \leq c & \left(\int_{T} \ldots \int_{T}\left|f\left(x_{1}, \ldots, x_{p}\right)\right|^{\alpha}\right. \\
& \left.\left(1+\log _{+} \frac{\left|f\left(x_{1}, \ldots, x_{p}\right)\right|^{2}}{\prod_{i=1}^{p} \psi\left(x_{i}\right)^{2 / \alpha}}\right)^{p-r-K-1} m\left(d x_{1}\right) \ldots m\left(d x_{p}\right)\right)^{\alpha / 2}<\infty .
\end{aligned}
$$

Another application of Proposition 5.1 of Samorodnitsky and Szulga (1989) shows that

$$
\begin{aligned}
& E S_{12}^{\alpha / 2} \leq c E\left(X^{\alpha / 2}\left(1+\log _{+} X\right)^{p-r-K}\right) \\
& \leq c \int_{S^{p}}\left|f\left(x_{1}, \ldots, x_{p}\right)\right|^{\alpha}\left(1+\log _{+} \frac{\left|f\left(x_{1}, \ldots, x_{p}\right)\right|^{2}}{\prod_{i=1}^{p} \psi\left(x_{i}\right)^{2 / \alpha}}\right)^{p-r-K} m\left(d x_{1}\right) \ldots m\left(d x_{p}\right)<\infty .
\end{aligned}
$$

The relations $(3.25),(3.26)$ and $(3.27)$ now establish that $E S_{1, K}^{\alpha / 2}<\infty$, and so we have completed the inductive argument, thus proving (3.22) and, hence, (3.21) as well. 
Having obtained (3.21) we immediately see that (3.17) holds as a particular case of (3.21) with $J=0$. Therefore, (3.4) has been completely proved.

Now we will prove (3.6). Observe that

$$
R_{r}=\sum_{r_{0}=0}^{r-1} c\left(r_{0}\right) R_{r, r_{0}}
$$

for some constants $c\left(r_{0}\right), r_{0}=0, \ldots, r-1$, where

$$
\begin{gathered}
R_{r, r_{0}}=\left[\prod_{i=1}^{r_{0}} \Gamma_{i}^{-2 / \alpha} \psi\left(V_{i}\right)^{-2 / \alpha}\right] \\
\sum_{m_{1}=r_{0}+2}^{\infty} \sum_{m_{2}=m_{1}+1}^{\infty} \ldots \sum_{m_{r-r_{0}}=m_{r-r_{0}-1}+1}^{\infty}\left[\prod_{d=1}^{r-r_{0}} \Gamma_{m_{d}}^{-2 / \alpha} \psi\left(V_{m_{d}}\right)^{-2 / \alpha}\right] \\
\sum_{\substack{\mathbf{j} \in D^{p+q-2 r}, j_{k}+r_{0} \neq m_{d} \\
k=1, \ldots, p+q-2 r, d=1, \ldots r-r_{0}}}^{p}\left[\prod_{k=1}^{p+q-2 r} \epsilon_{j_{k}+r_{0}} \Gamma_{j_{k}+r_{0}}^{-1 / \alpha} \psi\left(V_{j_{k}+r_{0}}\right)^{-1 / \alpha}\right] \\
h_{r}\left(V_{1}, \ldots, V_{r_{0}}, V_{m_{1}}, \ldots, V_{m_{r-r_{0}}}, V_{j_{1}+r_{0}}, \ldots, V_{j_{p+q-2 r}+r_{0}}\right)
\end{gathered}
$$

$r_{0}=0, \ldots, r-1$. That is, $(3.6)$ will follow once we check that for every such $r_{0}$

$$
\lim _{\lambda \rightarrow \infty} \lambda^{\alpha / 2}(\log \lambda)^{-(r-1)} P\left(\left|R_{r, r_{0}}\right|>\lambda\right)=0
$$

We start with "decoupling" $R_{r, r-0}$. We know by Theorem 1 of de la Pena and Montgomery-Smith (1995) that for some constant $c>0$ one has

$$
P\left(\left|R_{r, r_{0}}\right|>\lambda\right) \leq c P\left(\left|R_{r, r_{0}}^{(1)}\right|>\lambda / c\right)
$$

for all $\lambda>0$, where

$$
\begin{gathered}
R_{r, r_{0}}^{(1)}=\left[\prod_{i=1}^{r_{0}} \Gamma_{i}^{-2 / \alpha} \psi\left(V_{i}\right)^{-2 / \alpha}\right] \\
\sum_{m_{1}=r_{0}+2}^{\infty} \sum_{m_{2}=m_{1}+1}^{\infty} \ldots \sum_{\substack{\mathbf{j} \in D^{p+q}+2 r, j_{k}+r_{0} \neq m_{d} \\
m_{r-r_{0}}=m_{r-r_{0}-1}+1}}^{\infty}\left[\prod_{d=1}^{r-r_{0}} \Gamma_{m_{d}}^{-2 / \alpha} \psi\left(V_{m_{d}}^{(d)}\right)^{-2 / \alpha}\right] \\
\left.\sum_{k=1, \ldots, q-2 r, d=1, \ldots r-r_{0}}^{p+q-2 r} \epsilon_{j_{k}+r_{0}}^{(k)} \Gamma_{j_{k}+r_{0}}^{-1 / \alpha} \psi\left(V_{j_{k}+r_{0}}^{\left(r-r_{0}+k\right)}\right)^{-1 / \alpha}\right]
\end{gathered}
$$




$$
h_{r}\left(V_{1}, \ldots, V_{r_{0}}, V_{m_{1}}^{(1)}, \ldots, V_{m_{r-r_{0}}}^{\left(r-r_{0}\right)}, V_{j_{1}+r_{0}}^{\left(r-r_{0}+1\right)}, \ldots, V_{j_{p+q-2 r}+r_{0}}^{\left(p+q-r-r_{0}\right)}\right)
$$

$r_{0}=0, \ldots, r-1$. Here $\left(V_{j}^{(m)}, j \geq 1\right), m=1, \ldots, p+q-r-r_{0}$ are i.i.d. copies of $\left(V_{j}, j \geq 1\right)$ independent of i.i.d. copies $\left(\epsilon_{j}^{(m)}, j \geq 1\right), m=1, \ldots, p+q-2 r$ of $\left(\epsilon_{j}, j \geq\right.$ 1). Furthermore all these new sequences of random variables are independent of the sequences $\left(\Gamma_{j}, j \geq 1\right)$ and $\left(V_{j}, j \geq 1\right)$. Applying now the contraction principle for Rademacher series (e.g. Theorem 3.4. in Ledoux and Talagrand (1991)) we conclude further that there is a constant $c>0$ such that

$$
P\left(\left|R_{r, r_{0}}^{(1)}\right|>\lambda\right) \leq c P\left(\left|R_{r, r_{0}}^{(2)}\right|>\lambda / c\right)
$$

for all $\lambda>0$, where

$$
\begin{aligned}
& R_{r, r_{0}}^{(2)}=\left[\prod_{i=1}^{r_{0}} \Gamma_{i}^{-2 / \alpha} \psi\left(V_{i}\right)^{-2 / \alpha}\right] \\
& \sum_{m_{1}=r_{0}+2}^{\infty} \sum_{m_{2}=m_{1}+1}^{\infty} \ldots \sum_{m_{r-r_{0}}=m_{r-r_{0}-1}+1}^{\infty}\left[\prod_{d=1}^{r-r_{0}} \Gamma_{m_{d}}^{-2 / \alpha} \psi\left(V_{m_{d}}^{(d)}\right)^{-2 / \alpha}\right] \\
& \sum_{\substack{\mathbf{j} \in D^{p+q}-2 r, j_{k}+r_{0} \neq m_{d} \\
k=1, \ldots, p+q-2 r, d=1, \ldots r-r_{0}}}\left[\prod_{k=1}^{p+q-2 r} \epsilon_{j_{k}}^{(k)}\left(\Gamma_{j_{k}}^{(1)}\right)^{-1 / \alpha} \psi\left(V_{j_{k}}^{\left(r-r_{0}+k\right)}\right)^{-1 / \alpha}\right] \\
& h_{r}\left(V_{1}, \ldots, V_{r_{0}}, V_{m_{1}}^{(1)}, \ldots, V_{m_{r-r_{0}}}^{\left(r-r_{0}\right)}, V_{j_{1}}^{\left(r-r_{0}+1\right)}, \ldots, V_{j_{p+q-2 r}}^{\left(p+q-r-r_{0}\right)}\right) \text {, }
\end{aligned}
$$

$r_{0}=0, \ldots, r-1$, where the new sequence of random variables, $\left(\Gamma_{j}^{(1)}, j \geq 1\right)$ is a copy of $\left(\Gamma_{j}, j \geq 1\right)$ and is independent of all other random variables around. It follows from (3.30) and (3.31) that (3.6) will follow once we prove that

$$
\lim _{\lambda \rightarrow \infty} \lambda^{\alpha / 2}(\log \lambda)^{-(r-1)} P\left(\left|R_{r, r_{0}}^{(2)}\right|>\lambda\right)=0
$$

for every $r_{0}=0, \ldots r-1$.

We start by proving a similar statement for a "truncated" version of $R_{r, r_{0}}^{(2)}$. For an $M \geq 1$ define

$$
R_{r, r_{0}}^{(2)}(M)=\left[\prod_{i=1}^{r_{0}} \Gamma_{i}^{-2 / \alpha} \psi\left(V_{i}\right)^{-2 / \alpha}\right]
$$




$$
\begin{gathered}
\sum_{m_{1}=M}^{\infty} \sum_{m_{2}=m_{1}+1}^{\infty} \ldots \sum_{m_{r-r_{0}}=m_{r-r_{0}-1}+1}^{\infty}\left[\prod_{d=1}^{r-r_{0}} \Gamma_{m_{d}}^{-2 / \alpha} \psi\left(V_{m_{d}}^{(d)}\right)^{-2 / \alpha}\right] \\
\sum_{\substack{\mathbf{j} \in D^{p+q-2 r}, j_{k} \geq M, j_{k}+r_{0} \neq m_{d} \\
k=1, \ldots, p+q-2 r, d=1, \ldots r-r_{0}}}^{p+q-2 r}\left[\prod_{k=1}^{p+q} \epsilon_{j_{k}}^{(k)}\left(\Gamma_{j_{k}}^{(1)}\right)^{-1 / \alpha} \psi\left(V_{j_{k}}^{\left(r-r_{0}+k\right)}\right)^{-1 / \alpha}\right] \\
h_{r}\left(V_{1}, \ldots, V_{r_{0}}, V_{m_{1}}^{(1)}, \ldots, V_{m_{r-r_{0}}}^{\left(r-r_{0}\right)}, V_{j_{1}}^{\left(r-r_{0}+1\right)}, \ldots, V_{j_{p+q-2 r}}^{\left(p+q-r-r_{0}\right)}\right) .
\end{gathered}
$$

We claim that for all $M$ big enough,

$$
\lim _{\lambda \rightarrow \infty} \lambda^{\alpha / 2}(\log \lambda)^{-(r-1)} P\left(\left|R_{r, r_{0}}^{(2)}(M)\right|>\lambda\right)=0
$$

To proceed, we need the following lemma.

Lemma 3.3. Let $Z$ be a nonnegative random variable defined on the same probability space as the first $r$ points $\Gamma_{1}, \ldots, \Gamma_{r}, r \geq 1$, of a unit rate Poisson process on $(0, \infty)$. Assume that there is a $K<\infty$ such that

$$
E\left(Z \mid \Gamma_{1}, \ldots, \Gamma_{r}\right) \leq K \text { a.s. }
$$

Then

$$
\underset{t \downarrow 0}{\limsup t^{-1}}\left(\log \frac{1}{t}\right)^{-(r-1)} P\left(\prod_{i=1}^{r} \Gamma_{i} \leq t Z\right)<\infty
$$

Proof of Lemma 3.3. Let $F_{r}$ stand for the law of the product $\prod_{i=1}^{r} \Gamma_{i}$. We have by the Markov inequality

$$
\begin{aligned}
P\left(\prod_{i=1}^{r} \Gamma_{i} \leq t Z\right) & \leq P\left(\prod_{i=1}^{r} \Gamma_{i} \leq t\right)+K t \int_{t}^{\infty} y^{-1} F_{r}(d y) \\
& \leq P\left(\prod_{i=1}^{r} \Gamma_{i} \leq t\right)+K t \int_{t}^{\infty} \frac{P\left(\prod_{i=1}^{r} \Gamma_{i} \leq x\right)}{x^{2}} d x
\end{aligned}
$$

and (3.35) follows immediately from the fact that

$$
\lim _{t \downarrow 0} t^{-1}\left(\log \frac{1}{t}\right)^{-(r-1)} P\left(\prod_{i=1}^{r} \Gamma_{i} \leq t\right)<\infty
$$


(see e.g. Lemma 3.1 of Samorodnitsky and Szulga (1989)).

Back, once again, to the proof of Theorem 3.1, we notice that in order to use Lemma 3.3 to prove (3.33) it is enough to show that for all $M$ big enough,

$$
E\left|R_{r, r_{0}}^{(3)}(M)\right|^{\alpha / 2}<\infty
$$

where

$$
\begin{gathered}
R_{r, r_{0}}^{(3)}(M)=\prod_{i=1}^{r_{0}} \psi\left(V_{i}\right)^{-2 / \alpha} \\
\sum_{m_{1}=M}^{\infty} \sum_{m_{2}=m_{1}+1}^{\infty} \ldots \sum_{\substack{\mathbf{j} \in D^{p+q-2 r}, j_{k} \geq M, j_{k}+r_{0} \neq m_{d} \\
k=1, \ldots, p+q-2 r, d=1, \ldots r-r_{0}}}^{\infty}\left[\prod_{\substack{m_{r-r_{0}}=m_{r-r_{0}-1}+1 \\
h_{r}\left(V_{1}, \ldots, V_{r_{0}}, V_{m_{1}}^{(1)}, \ldots, V_{m_{r-r_{0}}}^{\left(r-r_{0}\right)}, V_{j_{1}}^{\left(r-r_{0}+1\right)}, \ldots, V_{j_{p+q-2 r}}^{\left(p+q-r-r_{0}\right)}\right) .}}^{p+q-2 r} \epsilon_{j_{k}}^{(k)}\left(\Gamma_{j_{k}}^{(1)}\right)^{-1 / \alpha} \psi\left(V_{j_{k}}^{\left(r-r_{0}+k\right)}\right)^{-1 / \alpha}\right]
\end{gathered}
$$

Using first the Khinchine inequality for tetrahedral sums in Rademacher random variables and then twice the Cauchy-Schwartz inequality we have

$$
\begin{aligned}
& E\left|R_{r, r_{0}}^{(3)}(M)\right|^{\alpha / 2} \\
& \leq c E\left[\prod_{i=1}^{r_{0}} \psi\left(V_{i}\right)^{-4 / \alpha} \sum_{\substack{\mathbf{j} \in D^{p+q-2 r} \\
j_{k} \geq M, k=1, \ldots, p+q-2 r}}\left[\prod_{k=1}^{p+q-2 r}\left(\Gamma_{j_{k}}^{(1)}\right)^{-2 / \alpha} \psi\left(V_{j_{k}}^{\left(r-r_{0}+k\right)}\right)^{-2 / \alpha}\right]\right. \\
& \left(\sum_{\substack{\mathbf{m} \in D^{r-r_{0}} \\
m_{d} \geq M, d=1, \ldots, r-r_{0}}}\left[\prod_{d=1}^{r-r_{0}} \Gamma_{m_{d}-r_{0}}^{-2 / \alpha} \psi\left(V_{m_{d}}^{(d)}\right)^{-2 / \alpha}\right]\right. \\
& \left.\left.h_{r}\left(V_{1}, \ldots, V_{r_{0}}, V_{m_{1}}^{(1)}, \ldots, V_{m_{r-r_{0}}}^{\left(r-r_{0}\right)}, V_{j_{1}}^{\left(r-r_{0}+1\right)}, \ldots, V_{j_{p+q-2 r}}^{\left(p+q-r-r_{0}\right)}\right)\right)^{2}\right]^{\alpha / 4} \\
& \leq c E\left[\prod _ { i = 1 } ^ { r _ { 0 } } \psi ( V _ { i } ) ^ { - 1 } \left(\sum_{\substack{\mathbf{j} \in D^{p+q}-2 r \\
j_{k} \geq M, k=1, \ldots, p+q-2 r}}\left[\prod_{k=1}^{p+q-2 r}\left(\Gamma_{j_{k}}^{(1)}\right)^{-2 / \alpha} \psi\left(V_{j_{k}}^{\left(r-r_{0}+k\right)}\right)^{-2 / \alpha}\right]\right.\right.
\end{aligned}
$$




$$
\begin{aligned}
& \left(\sum_{\substack{\mathbf{m} \in D^{r-r_{0}} \\
m_{d} \geq M, d=1, \ldots, r-r_{\mathbf{0}}}}\left[\prod_{d=1}^{r-r_{0}} \Gamma_{m_{d}-r_{0}}^{-2 / \alpha} \psi\left(V_{m_{d}}^{(d)}\right)^{-2 / \alpha}\right]\right. \\
& \left.f\left(V_{1}, \ldots, V_{r_{0}}, V_{m_{1}}^{(1)}, \ldots, V_{m_{r-r_{0}}}^{\left(r-r_{0}\right)}, V_{j_{1}}^{\left(r-r_{0}+1\right)}, \ldots, V_{j_{p-r}}^{\left(p-r_{0}\right)}\right)\right)^{2} \\
& \left(\sum_{\substack{\mathbf{m} \in D^{r-r_{0}} \\
m_{d} \geq M, d=1, \ldots, r-r_{0}}}\left[\prod_{d=1}^{r-r_{0}} \Gamma_{m_{d}-r_{0}}^{-2 / \alpha} \psi\left(V_{m_{d}}^{(d)}\right)^{-2 / \alpha}\right]\right. \\
& \left.\left.\left.g\left(V_{1}, \ldots, V_{r_{0}}, V_{m_{1}}^{(1)}, \ldots, V_{m_{r-r_{0}}}^{\left(r-r_{0}\right)}, V_{j_{p-r+1}}^{\left(p-r_{0}+1\right)}, \ldots, V_{j_{p+q-2 r}}^{\left(p+q-r-r_{0}\right)}\right)\right)^{2}\right)^{\alpha / 4}\right] \\
& \leq c\left\{E \left[\prod _ { i = 1 } ^ { r _ { 0 } } \psi ( V _ { i } ) ^ { - 1 } \left(\sum_{\substack{\mathbf{j} \in D^{p-r} \\
j_{k} \geq M, k=1, \ldots, p-r}}\left[\prod_{k=1}^{p-r}\left(\Gamma_{j_{k}}^{(1)}\right)^{-2 / \alpha} \psi\left(V_{j_{k}}^{\left(r-r_{0}+k\right)}\right)^{-2 / \alpha}\right]\right.\right.\right. \\
& \left(\sum_{\substack{\mathbf{m} \in D^{r-r_{0}} \\
m_{d} \geq M, d=1, \ldots, r-r_{0}}}\left[\prod_{d=1}^{r-r_{0}} \Gamma_{m_{d}-r_{0}}^{-2 / \alpha} \psi\left(V_{m_{d}}^{(d)}\right)^{-2 / \alpha}\right]\right. \\
& \left.\left.\left.\left.f\left(V_{1}, \ldots, V_{r_{0}}, V_{m_{1}}^{(1)}, \ldots, V_{m_{r-r_{0}}}^{\left(r-r_{0}\right)}, V_{j_{1}}^{\left(r-r_{0}+1\right)}, \ldots, V_{j_{p-r}}^{\left(p-r_{0}\right)}\right)\right)^{2}\right)^{\alpha / 2}\right]\right\}^{1 / 2} \\
& \left\{E \left[\prod _ { i = 1 } ^ { r _ { 0 } } \psi ( V _ { i } ) ^ { - 1 } \left(\sum_{\substack{\mathbf{j} \in D^{q-r} \\
j_{k} \geq M, k=1, \ldots, q-r}}\left[\prod_{k=1}^{q-r}\left(\Gamma_{j_{k}}^{(1)}\right)^{-2 / \alpha} \psi\left(V_{j_{k}}^{\left(r-r_{0}+k\right)}\right)^{-2 / \alpha}\right]\right.\right.\right. \\
& \left(\sum_{\substack{\mathbf{m} \in D^{r-r_{0}} \\
m_{d} \geq M, d=1, \ldots, r-r_{0}}}\left[\prod_{d=1}^{r-r_{0}} \Gamma_{m_{d}-r_{0}}^{-2 / \alpha} \psi\left(V_{m_{d}}^{(d)}\right)^{-2 / \alpha}\right]\right. \\
& \left.\left.\left.\left.g\left(V_{1}, \ldots, V_{r_{0}}, V_{m_{1}}^{(1)}, \ldots, V_{m_{r-r_{0}}}^{\left(r-r_{0}\right)}, V_{j_{1}}^{\left(r-r_{0}+1\right)}, \ldots, V_{j_{q-r}}^{\left(q-r_{0}\right)}\right)\right)^{2}\right)^{\alpha / 2}\right]\right\}^{1 / 2}
\end{aligned}
$$

Since both expectations above are of the same nature, our statement (3.36) will follow once we prove that

$$
E\left[\prod _ { i = 1 } ^ { r _ { 0 } } \psi ( V _ { i } ) ^ { - 1 } \left(\sum_{\substack{\mathbf{j} \in D^{p-r} \\ j_{k} \geq M, k=1, \ldots, p-r}}\left[\prod_{k=1}^{p-r}\left(\Gamma_{j_{k}}^{(1)}\right)^{-2 / \alpha} \psi\left(V_{j_{k}}^{\left(r-r_{0}+k\right)}\right)^{-2 / \alpha}\right]\right.\right.
$$




$$
\begin{gathered}
\left(\sum_{\substack{m \in D^{r-r_{0}} \\
m_{d} \geq M, d=1, \ldots, r-r_{0}}}\left[\prod_{d=1}^{r-r_{0}} \Gamma_{m_{d}-r_{0}}^{-2 / \alpha} \psi\left(V_{m_{d}}^{(d)}\right)^{-2 / \alpha}\right]\right. \\
\left.\left.\left.f\left(V_{1}, \ldots, V_{r_{0}}, V_{m_{1}}^{(1)}, \ldots, V_{m_{r-r_{0}}}^{\left(r-r_{0}\right)}, V_{j_{1}}^{\left(r-r_{0}+1\right)}, \ldots, V_{j_{p-r}}^{\left(p-r_{0}\right)}\right)\right)^{2}\right)^{\alpha / 2}\right]<\infty .
\end{gathered}
$$

However, the fact that for $M$ large enough (3.37) follows from $N_{p}(f)<\infty$ can be established by exactly the same argument as that used in proving (3.21). Hence, (3.33) follows.

Recall that (3.33) is a statement analogous to (3.32) but for a "truncated" version of $R_{r, r_{0}}^{(2)}$. Our next step is to prove another such statement, this time for a "less truncated" version of $R_{r, r_{0}}^{(2)}$. Specifically, we claim that for all $M$ big enough,

$$
\lim _{\lambda \rightarrow \infty} \lambda^{\alpha / 2}(\log \lambda)^{-(r-1)} P\left(\left|R_{r, r_{0}}^{(4)}(M)\right|>\lambda\right)=0
$$

where for $M \geq 1$

$$
\begin{aligned}
& R_{r, r_{0}}^{(4)}(M)=\left[\prod_{i=1}^{r_{0}} \Gamma_{i}^{-2 / \alpha} \psi\left(V_{i}\right)^{-2 / \alpha}\right] \\
& \sum_{m_{1}=r_{0}+2}^{\infty} \sum_{m_{2}=m_{1}+1}^{\infty} \ldots \sum_{m_{r-r_{0}}=m_{r-r_{0}-1}+1}^{\infty}\left[\prod_{d=1}^{r-r_{0}} \Gamma_{m_{d}}^{-2 / \alpha} \psi\left(V_{m_{d}}^{(d)}\right)^{-2 / \alpha}\right] \\
& \sum_{\substack{\mathbf{j} \in D^{p+q}-2 r \\
k=1, j_{k} \geq M, j_{k}+r_{0} \neq m_{d} \\
k=p+q, 2 r, d=1, \ldots r-r_{0}}}\left[\prod_{k=1}^{p+q-2 r} \epsilon_{j_{k}}^{(k)}\left(\Gamma_{j_{k}}^{(1)}\right)^{-1 / \alpha} \psi\left(V_{j_{k}}^{\left(r-r_{0}+k\right)}\right)^{-1 / \alpha}\right] \\
& h_{r}\left(V_{1}, \ldots, V_{r_{0}}, V_{m_{1}}^{(1)}, \ldots, V_{m_{r-r_{0}}}^{\left(r-r_{0}\right)}, V_{j_{1}}^{\left(r-r_{0}+1\right)}, \ldots, V_{j_{p+q-2 r}}^{\left(p+q-r-r_{0}\right)}\right) .
\end{aligned}
$$

Observe that for all $M \geq r_{0}+2$,

$$
R_{r, r_{0}}^{(4)}(M)=R_{r, r_{0}}^{(2)}(M)+\sum_{n=r_{0}+2}^{M-1} R_{r, r_{0}}(M, n)
$$

where for $n=r_{0}+2, \ldots, M-1$

$$
R_{r, r_{0}}(M, n)=\left[\prod_{i=1}^{r_{0}} \Gamma_{i}^{-2 / \alpha} \psi\left(V_{i}\right)^{-2 / \alpha}\right] \Gamma_{n}^{-2 / \alpha} \psi\left(V_{n}^{(1)}\right)^{-2 / \alpha}
$$




$$
\begin{gathered}
\sum_{m_{2}=n+1}^{\infty} \ldots \sum_{\substack{m_{r-r_{0}}=m_{r-r_{0}-1}+1 \\
\mathbf{j} \in D^{p+q-2 r}, j_{k} \geq M, j_{k}+r_{0} \neq m_{d} \\
k=1, \ldots, p+q-2 r, d=1, \ldots r-r_{0}}}^{\infty}\left[\prod_{k=1}^{r-r_{0}} \Gamma_{m_{d}}^{-2 / \alpha} \psi\left(V_{m_{d}}^{(d)}\right)^{-2 / \alpha}\right] \\
h_{r}\left(V_{1}, \ldots, V_{r_{0}}, V_{n}^{(1)}, V_{m_{2}}^{(2)}, \ldots, V_{m_{r-r_{0}}}^{\left(r-r_{0}\right)}, V_{j_{1}}^{\left(r-r_{0}+1\right)}, \ldots, V_{j_{p+q-2 r}}^{\left(p+q-r-r_{0}\right)}\right) .
\end{gathered}
$$

Since (3.33) holds for $M$ big enough, (3.38) will follow once we prove that for all $M$ big enough and all $n=r_{0}+2, \ldots, M-1$

$$
\lim _{\lambda \rightarrow \infty} \lambda^{\alpha / 2}(\log \lambda)^{-(r-1)} P\left(\left|R_{r, r_{0}}(M, n)\right|>\lambda\right)=0
$$

Applying the same downward induction as the one used in the proof of (3.21) we see that (3.39) will follow if we prove that for all $M$ large enough,

$$
\lim _{\lambda \rightarrow \infty} \lambda^{\alpha / 2}(\log \lambda)^{-(r-1)} P\left(\left|R_{r, r_{0}}^{(5)}(M)\right|>\lambda\right)=0
$$

where

$$
\begin{gathered}
R_{r, r_{0}}^{(5)}(M, n)=\left[\prod_{i_{1}=1}^{r_{0}} \Gamma_{i_{1}}^{-2 / \alpha} \psi\left(V_{i_{1}}\right)^{-2 / \alpha}\right]\left[\prod_{i_{2}=r_{0}+2}^{r-1} \Gamma_{i_{2}}^{-2 / \alpha} \psi\left(V_{i_{2}}^{\left(i_{2}-r_{0}+1\right)}\right)^{-2 / \alpha}\right] \\
\sum_{\substack{j \in D^{p+q-2 r} \\
j_{k} \geq M, k=1, \ldots, p+q-2 r}}\left[\prod_{k=1}^{p+q-2 r} \epsilon_{j_{k}}^{(k)}\left(\Gamma_{j_{k}}^{(1)}\right)^{-1 / \alpha} \psi\left(V_{j_{k}}^{\left(r-r_{0}+k\right)}\right)^{-1 / \alpha}\right] \\
h_{r}\left(V_{1}, \ldots, V_{r_{0}}, V_{r_{0}+2}^{(1)}, \ldots, V_{r+1}^{\left(r-r_{0}\right)}, V_{j_{1}}^{\left(r-r_{0}+1\right)}, \ldots, V_{j_{p+q-2 r}}^{\left(p+q-r-r_{0}\right)}\right) .
\end{gathered}
$$

However, the fact that (3.40) holds for all $M$ large enough follows immediately from the fact that (3.33) holds for all $M$ large enough. Therefore, (3.38) follows.

We are now in a position to complete the proof of (3.32). Fix an $M$ for which (3.38) holds. It is clear that (3.32) will follow once we establish that for every $n=1, M-1$

$$
\lim _{\lambda \rightarrow \infty} \lambda^{\alpha / 2}(\log \lambda)^{-(r-1)} P\left(\left|R_{r, r_{0}}^{(6)}(n)\right|>\lambda\right)=0
$$


where

$$
\begin{aligned}
& R_{r, r_{0}}^{(6)}(n)=\left[\prod_{i=1}^{r_{0}} \Gamma_{i}^{-2 / \alpha} \psi\left(V_{i}\right)^{-2 / \alpha}\right]\left(\Gamma_{n}^{(1)}\right)^{-1 / \alpha} \psi\left(V_{n}^{\left(r-r_{0}+1\right)}\right)^{-1 / \alpha} \\
& \sum_{\substack{m_{1} \geq r_{0}+2 \\
m_{1} \neq n}} \sum_{\substack{m_{2} \geq m_{1}+1 \\
m_{2} \neq n}} \cdots \sum_{\substack{m_{r-r_{0}} \geq m_{r}-r_{0}-1 \\
m_{r-r_{0} \neq n} \neq n}}\left[\prod_{d=1}^{r-r_{0}} \Gamma_{m_{d}}^{-2 / \alpha} \psi\left(V_{m_{d}}^{(d)}\right)^{-2 / \alpha}\right] \\
& \sum_{\substack{\mathbf{j} \in D^{p+q}-2 r-1, j_{k}>n, j_{k}+r_{0} \neq m_{d} \\
k=1, \ldots, p+q-2 r-1, d=1, \ldots r-r_{\mathbf{0}}}}\left[\prod_{k=1}^{p+q-2 r-1} \epsilon_{j_{k}}^{(k)}\left(\Gamma_{j_{k}}^{(1)}\right)^{-1 / \alpha} \psi\left(V_{j_{k}}^{\left(r-r_{0}+k+1\right)}\right)^{-1 / \alpha}\right] \\
& h_{r}\left(V_{1}, \ldots, V_{r_{0}}, V_{m_{1}}^{(1)}, \ldots, V_{m_{r-r_{0}}}^{\left(r-r_{0}\right)}, V_{n}^{\left(r-r_{0}+1\right)}, V_{j_{2}}^{\left(r-r_{0}+1\right)}, \ldots, V_{j_{p+q-2 r-1}}^{\left(p+q-r-r_{0}\right)}\right) .
\end{aligned}
$$

Repeating the truncation and downward induction argument used a number of times before, we see that (3.41) will be a consequence of the following statement.

$$
\lim _{\lambda \rightarrow \infty} \lambda^{\alpha / 2}(\log \lambda)^{-(r-1)} P\left(\left|R_{r, r_{0}}^{(7)}\right|>\lambda\right)=0
$$

where

$$
\begin{gathered}
R_{r, r_{0}}^{(7)}=\prod_{i_{1}=1}^{r_{0}} \Gamma_{i_{1}}^{-2 / \alpha} \prod_{i_{2}=r_{0}+2}^{r+1} \Gamma_{i_{2}}^{-2 / \alpha} \prod_{j=1}^{p+q-2 r}\left(\Gamma_{j}^{(1)}\right)^{-1 / \alpha} \\
\prod_{k_{1}=1}^{r} \psi\left(V_{k_{1}}\right)^{-2 / \alpha} \prod_{k_{2}=r+1}^{p+q-r} \psi\left(V_{k_{2}}\right)^{-1 / \alpha} \\
f\left(V_{1}, \ldots, V_{r}, V_{r+1}, \ldots, V_{p}\right) g\left(V_{1}, \ldots, V_{r}, V_{p+1}, \ldots, V_{p+q-r}\right) .
\end{gathered}
$$

We need yet another lemma.

Lemma 3.4. Let $Z$ be a nonnegative random variable independent of a sequence $\left(\Gamma_{j}, j \geq 1\right)$ of the ordered points of a unit rate Poisson process on $(0, \infty)$, with $E Z<\infty$. Then for every $r \geq 1$,

$$
\lim _{t \downarrow 0} t^{-1}\left(\log \frac{1}{t}\right)^{-(r-1)} P\left(\left(\prod_{i=1}^{r-1} \Gamma_{i}\right) \Gamma_{r+1} \leq t Z\right)=0 .
$$

Proof of Lemma 3.4. Let $\Gamma_{2}^{(1)}$ be a copy of $\Gamma_{2}$ independent both of the original Poisson points and $Z$. We have

$$
P\left(\left(\prod_{i=1}^{r-1} \Gamma_{i}\right) \Gamma_{r+1} \leq t Z\right) \leq P\left(\left(\prod_{i=1}^{r-1} \Gamma_{i}\right) \leq t \frac{Z}{\Gamma_{2}^{(1)}}\right)
$$




$$
\leq O\left(t\left(\log \frac{1}{t}\right)^{r-2}\right)
$$

by Lemma 3.3 since $E\left(\Gamma_{2}\right)^{-1}<\infty$.

We now complete the proof of Theorem 3.1. It follows from Lemma 3.4 that (3.42) will be established if

$$
\begin{gathered}
E \mid \prod_{k_{1}=1}^{r} \psi\left(V_{k_{1}}\right)^{-2 / \alpha} \prod_{k_{2}=r+1}^{p+q-r} \psi\left(V_{k_{2}}\right)^{-1 / \alpha} f\left(V_{1}, \ldots, V_{r}, V_{r+1}, \ldots, V_{p}\right) \\
\left.g\left(V_{1}, \ldots, V_{r}, V_{p+1}, \ldots, V_{p+q-r}\right)\right|^{\alpha / 2}<\infty .
\end{gathered}
$$

However, (3.44) follows from $N_{p}(f)<\infty$ and $N_{q}(g)<\infty$ by Hölder's inequality. This proves (3.41), and hence (3.32). Therefore, the proof of Theorem 3.1 is now complete.

\section{Tail of the product and independence of multiple stable integrals.}

Theorems 2.3 and 3.1 allow us to determine the asymptotic behavior of the tail of the product of two multiple stable integrals.

Theorem 4.1. Assume $N_{p}(f)<\infty$ and $N_{q}(g)<\infty$, where $f: S^{p} \rightarrow \mathbf{R}$ and $g: S^{q} \rightarrow \mathbf{R}$ are symmetric vanishing on diagonals functions. Let $r=\max \{i$ : $\left.C_{i}(f, g) \neq 0\right\}$, where $C_{i}(f, g)$ is given by (3.2) and $C_{0}(f, g)=\|\widehat{f \otimes g}\|_{L^{\alpha}\left(S^{q}\right)}^{\alpha}$. Then, as $\lambda \rightarrow \infty$,

$$
P\left(\left|I_{p}^{M}(f) I_{q}^{M}(g)\right|>\lambda\right) \sim \begin{cases}k_{0, \alpha} \lambda^{-\alpha}(\ln \lambda)^{p+q-1} C_{0}(f, g) & \text { if } r=0 \\ k_{r, \alpha} \lambda^{-\alpha / 2}(\ln \lambda)^{r-1} C_{r}(f, g) & \text { if } r=1, \ldots, p \wedge q,\end{cases}
$$

where $k_{r, \alpha}$ are finite numerical constants in (3.0) and (3.1).

Proof. The proof follows from Theorems 2.3 and 3.1 by a standard application of the inequality

$P(|X|>(1+\epsilon) \lambda)-P(|Y|>\epsilon \lambda) \leq P(|X+Y|>\lambda) \leq P(|X|>(1-\epsilon) \lambda)+P(|Y|>\epsilon \lambda)$ 
which is valid for any random variables $X$ and $Y, \epsilon \in(0,1)$, and $\lambda>0$.

Now we are ready to establish conditions for independence of $I_{p}^{M}(f)$ and $I_{q}^{M}(g)$. The following lemma will be used below and in Section 5. Its proof uses a method from Üstünel and Zakai (1989).

Lemma 4.2. Let $u: S^{k} \rightarrow \mathbf{R}$ and $v: S^{n} \rightarrow \mathbf{R}$ be measurable symmetric functions such that $u \in L^{2}\left(S^{k}, \nu^{\otimes k}\right)$ and $v \in L^{2}\left(S^{n}, \nu^{\otimes n}\right)$, where $\nu$ is a $\sigma$-finite measure on S. Then

$$
\|\widehat{u \otimes v}\|_{L^{2}\left(S^{k+n}\right)}^{2}=\frac{k ! n !}{(k+n) !}\|u \otimes v\|_{L^{2}\left(S^{k+n}\right)}^{2}+\sum_{i=1}^{k \wedge n} c_{i}\left\|u \otimes_{i} v\right\|_{L^{2}\left(S^{k+n-2 i}\right)}^{2}
$$

where

$$
\begin{aligned}
& =\int_{S^{i}} u\left(s_{1}, \ldots, s_{k-i}, x_{1}, \ldots, x_{i}\right) v\left(x_{1}, \ldots, x_{i}, s_{k-i+1}, \ldots, s_{k+n-2 i}\right) \nu\left(d x_{1}\right) \ldots \nu\left(d x_{i}\right) . \\
& \text { and } c_{i}=\left(\begin{array}{c}
k \\
i
\end{array}\right)\left(\begin{array}{c}
n \\
i
\end{array}\right) /\left(\begin{array}{c}
k+n \\
k
\end{array}\right) .
\end{aligned}
$$

Proof. We have

$$
\begin{gathered}
\|\widehat{u \otimes v}\|_{L^{2}\left(S^{k+n}\right)}^{2} \\
=((k+n) !)^{-2} \int_{S^{k+n}}\left|\sum_{\pi} u\left(s_{\pi_{1}}, \ldots, s_{\pi_{k}}\right) v\left(s_{\pi_{k+1}}, \ldots, s_{\pi_{k+n}}\right)\right|^{2} d \nu^{\otimes(k+n)} \\
=((k+n) !)^{-2} \int_{S^{k+n}} D_{\pi_{, \mu}}\left(s_{1}, \ldots, s_{k+n}\right) d \nu^{\otimes(k+n)},
\end{gathered}
$$

where $\pi, \mu$ run over all permutations of $1, \ldots, k+n$ and

$$
D_{\pi, \mu}=u\left(s_{\pi_{1}}, \ldots, s_{\pi_{k}}\right) v\left(s_{\mu_{k+1}}, \ldots, s_{\mu_{k+n}}\right) u\left(s_{\mu_{1}}, \ldots, s_{\mu_{k}}\right) v\left(s_{\pi_{k+1}}, \ldots, s_{\pi_{k+n}}\right) .
$$

Suppose that $k \leq n$ and consider the sets $J_{1}(\pi, \mu)=\left\{\pi_{1}, \ldots, \pi_{k}\right\} \cap\left\{\mu_{1}, \ldots, \mu_{k}\right\}$ and $J_{2}(\pi, \mu)=\left\{\pi_{k+1}, \ldots, \pi_{k+n}\right\} \cap\left\{\mu_{k+1}, \ldots, \mu_{k+n}\right\}$. If $\operatorname{Card}\left(J_{1}(\pi, \mu)\right)=k-i$, then $\operatorname{Card}\left(J_{2}(\pi, \mu)\right)=n+i, \quad i=0, \ldots, k$. If $i=0$ then $D_{\pi, \mu}=|u \otimes v|^{2}$. Suppose $i \geq 1$. Then by Fubini's theorem

$$
\int_{S^{k+n}} D_{\pi, \mu} d \nu^{k+n}=\left\|u \otimes_{i} v\right\|_{L^{2}\left(S^{k+n-2 i}\right)}^{2} .
$$

Since there are $\left(\begin{array}{c}k \\ i\end{array}\right)\left(\begin{array}{c}n \\ i\end{array}\right) k ! n !(k+n) ! \operatorname{terms} D_{\pi, \mu}$ with $\operatorname{Card}\left(J_{1}(\pi, \mu)\right)=k-i, i \geq 0$, the proof is complete. 
Theorem 4.3. Assume $N_{p}(f)<\infty$ and $N_{q}(g)<\infty$, where $f: S^{p} \rightarrow \mathbf{R}$ and $g: S^{q} \rightarrow \mathbf{R}$ are symmetric vanishing on diagonals functions. Then $I_{p}^{M}(f)$ and $I_{q}^{M}(g)$ are independent if and only if there exist disjoint measurable sets $A, B \subset S$ such that supp $\{f\} \subset A^{\otimes p} \operatorname{modulo} m^{p}$ and $\operatorname{supp}\{g\} \subset B^{q} \operatorname{modulo} m^{\otimes q}$.

Proof. Sufficiency. Since $\{M(E \cap A): E \in \mathcal{S}\}$ and $\{M(E \cap B): E \in \mathcal{S}\}$ are independent, $I_{p}^{M}(f)=I_{p}^{M}\left(f \mathbf{1}_{A^{p}}\right)$ and $I_{q}^{M}(g)=I_{q}^{M}\left(g \mathbf{1}_{B^{q}}\right)$ are independent.

Necessity. Since $I_{p}^{M}(f)$ and $I_{q}^{M}(g)$ are independent, the tail of $\left|I_{p}^{M}(f) I_{q}^{M}(g)\right|$ is of the order $\lambda^{-\alpha}(\log \lambda)^{p+q-1}$. From Theorem 4.1 we have $C_{1}(f, g)=\cdots=$ $C_{p \wedge q}(f, g)=0$. Hence for $r=1, \ldots, p \wedge q$

$$
\hat{h}_{r}\left(s_{1}, \ldots, s_{r}, \cdot\right)=0 \quad m^{\otimes(p+q-r)}-\text { a.e. }
$$

(see the definition of $\hat{h}_{r}$ in Theorem 3.1). Choose a sequence $s_{1}, \ldots, s_{p+q-1}$ of points from $S$ and define a measure $\nu=\sum_{i=2}^{p+q-1} \delta_{s_{i}}$. Consider the multiple integral

$$
L\left(s_{1}, \ldots, s_{p+q-1}\right):=\int_{S^{p+q-1}}\left|\hat{h}_{1}\left(s_{1}, x_{2}, \ldots, x_{p+q-1}\right)\right|^{2} \nu\left(d x_{2}\right) \cdots \nu\left(d x_{p+q-1}\right) .
$$

Notice that for each $s_{1}, h_{r}\left(s_{1}, \cdot\right)$ is a product of two symmetric measurable functions, so that from Lemma 4.2 we get

$$
\begin{aligned}
L\left(s_{1}, \ldots, s_{p+q-1}\right) & \geq \frac{p ! q !}{(p+q) !} \int_{S^{p-1}}\left|f\left(s_{1}, \cdot\right)\right|^{2} d \nu^{\otimes(p-1)} \int_{S^{q-1}}\left|g\left(s_{1}, \cdot\right)\right|^{2} d \nu^{\otimes(q-1)} \\
& \left.\geq \frac{p ! q !(p-1) !(q-1) !}{(p .5)} \mid f\left(s_{1}, \ldots, s_{p}\right)\right)\left.\right|^{2}\left|g\left(s_{1}, s_{p+1}, \ldots, s_{p+q-1}\right)\right|^{2} .
\end{aligned}
$$

Now observe that

$$
L\left(s_{1}, \ldots, s_{p+q-1}\right)=\sum_{r=1}^{p \wedge q} a_{r} \sum_{1=i_{1}<i_{2}<\cdots<i_{r} \leq p+q-1}\left|\hat{h}_{r}\left(s_{i_{1}}, \ldots, s_{i_{r}}, \cdot\right)\right|^{2},
$$

for some constants $a_{r} \geq 0$; the symmetrization in $\hat{h}_{r}\left(s_{i_{1}}, \ldots, s_{i_{r}}, \cdot\right)$ is taken with respect to variables $s_{j}$ with $j \in\{1, \ldots, p+q-1\} \backslash\left\{i_{1}, \ldots, i_{r}\right\}$. In view of (4.3), $L=0 \quad m^{\otimes(p+q-1)}$-almost everywhere; (4.5) implies that

$$
f\left(s_{1}, \ldots, s_{p}\right) g\left(s_{1}, s_{p+1}, \ldots, s_{p+q-1}\right)=0 \quad m^{\otimes(p+q-1)}-a . e .
$$


Consequently,

$$
\begin{gathered}
0=m^{\otimes(p+q-1)}\left\{\left(s_{1}, \ldots, s_{p+q-1}\right): f\left(s_{1}, \ldots, s_{p}\right) \neq 0, g\left(s_{1}, s_{p+1}, \ldots, s_{p+q-1}\right) \neq 0\right\} \\
=\int_{S} m^{\otimes(p-1)}\left\{\left(s_{2}, \ldots, s_{p}\right): f\left(s_{1}, \cdot\right) \neq 0\right\} \\
m^{\otimes(q-1)}\left\{\left(s_{p+1}, \ldots, s_{p+q-1}\right): g\left(s_{1}, \cdot\right) \neq 0\right\} m\left(d s_{1}\right) .
\end{gathered}
$$

Hence the sets

$$
A=\left\{s_{1}: m^{\otimes(p-1)}\left\{\left(s_{2}, \ldots, s_{p}\right): f\left(s_{1}, \cdot\right) \neq 0\right\}>0\right\}
$$

and

$$
B=\left\{s_{1}: m^{\otimes(q-1)}\left\{\left(s_{p+1}, \ldots, s_{p+q-1}\right): g\left(s_{1}, \cdot\right) \neq 0\right\}>0\right\}
$$

satisfy $m(A \cap B)=0$ and from the symmetry of $f$ and $g, \operatorname{supp}\{f\} \subset A^{p}$ modulo $m^{\otimes p}$ and $\operatorname{supp}\{g\} \subset B^{q}$ modulo $m^{\otimes q}$. Finally we may replace $A$ by $A \cap B^{c}$ to have $A$ and $B$ disjoint as in the statement of this theorem. The proof is complete.

\section{Independence of multiple integrals with respect to Gaussian and Poisson random measures.}

The purpose of this section is to exhibit the similarities and differences between the independence criteria for stable and Gaussian multiple integrals. We will also point out a gap in the discussion of independence of multiple Poisson integrals in Privault (1996).

Let $W$ be a Wiener random measure on $(S, \mathcal{S})$ with atomless $\sigma$-finite control measure $m$. Let $I_{p}^{W}(f)$ denote the p-tuple Wiener-Itô integral of $f \in L^{2}\left(S^{p}\right)(=$ $\left.L^{2}\left(S^{p}, m^{\otimes p}\right)\right)$ with respect to $W$. We refer the reader to Major (1980) for the necessary facts on Wiener-Itô integrals. In what follows, $f \in L^{2}\left(S^{p}\right), g \in L^{2}\left(S^{q}\right)$ are assumed to be symmetric and vanishing on the diagonals. $I_{p}^{W}(f)$ and $I_{q}^{W}(g)$ satisfy the product formula

$$
I_{p}^{W}(f) I_{q}^{W}(g)=\sum_{i=0}^{p \wedge q} i !\left(\begin{array}{c}
p \\
i
\end{array}\right)\left(\begin{array}{l}
q \\
i
\end{array}\right) I_{p+q-2 i}^{W}\left(f \otimes_{i} g\right)
$$


where $f \otimes_{i} g$ is defined analogously to $(4.2)$,

$$
f \otimes_{i} g\left(s_{1}, \ldots, s_{p+q-2 i}\right)
$$

$=\int_{S^{i}} f\left(s_{1}, \ldots, s_{k-i}, x_{1}, \ldots, x_{i}\right) g\left(x_{1}, \ldots, x_{i}, s_{k-i+1}, \ldots, s_{k+n-2 i}\right) m\left(d x_{1}\right) \ldots m\left(d x_{i}\right)$.

We have the isometry property $E\left[I_{k}^{W}(h)^{2}\right]=k !\|\hat{h}\|_{L^{2}\left(S^{k}\right)}^{2}$, where $\hat{h}$ denotes the symmetrization of $h$. In the proof of the following result, due to Üstünel and Zakai (1989), we follow their proof of the necessity part and that of Kallenberg (1991) for the sufficiency part.

Theorem 5.1. (Üstünel and Zakai (1989)) $I_{p}^{W}(f)$ and $I_{q}^{W}(g)$ are independent if and only if

$$
f \otimes_{1} g=0 \quad m^{\otimes(p+q-2)}-\text { a.e. }
$$

Proof. Necessity (Üstünel and Zakai (1989)). By the independence and the isometry property

$$
E\left[\left(I_{p}^{W}(f) I_{q}^{W}(g)\right)^{2}\right]=p !\|f\|_{L^{2}\left(S^{p}\right)} \cdot q !\|g\|_{L^{2}\left(S^{q}\right)}=p ! q !\|f \otimes g\|_{L^{2}\left(S^{p+q}\right)}
$$

Using product formula (5.1), orthogonality of multiple integrals of different orders, and the isometry property,

$$
E\left[\left(I_{p}^{W}(f) I_{q}^{W}(g)\right)^{2}\right]=\sum_{i=0}^{p \wedge q}\left[i !\left(\begin{array}{c}
p \\
i
\end{array}\right)\left(\begin{array}{l}
q \\
i
\end{array}\right)\right]^{2}(p+q-2 i) !\left\|\left(\widehat{f \otimes_{i} g}\right)\right\|_{L^{2}\left(S^{p+q-2 i}\right)}^{2}
$$

Dropping terms with $i \geq 1$ yields

$$
\begin{aligned}
E\left[\left(I_{p}^{W}(f) I_{q}^{W}(g)\right)^{2}\right] & \geq(p+q) !\|(\widehat{f \otimes g})\|_{L^{2}\left(S^{p+q}\right)}^{2} \\
& \geq p ! q !\|f \otimes g\|_{L^{2}\left(S^{p+q}\right)}^{2}+p ! q ! p q\left\|f \otimes_{1} g\right\|_{L^{2}\left(S^{p+q-2}\right)}^{2}
\end{aligned}
$$

where the last inequality uses Lemma 4.2. Now (5.3) and (5.4) give $f \otimes_{1} g=0$ $m^{\otimes(p+q-2)}-$ a.e., as claimed. 
Sufficiency (Kallenberg (1991)). Let $\left\{\phi_{n}\right\}$ be any orthonormal elements in $L^{2}(S)$ and let $H_{n}$ be the Hermite polynomial of degree $n$. By Itô formula (see Major (1980), Theorem 4.2)

$$
I_{p}^{W}\left(\phi_{1}^{\otimes p_{1}} \otimes \cdots \otimes \phi_{m}^{\otimes p_{m}}\right)=\prod_{k=1}^{m} H_{p_{k}}\left(I_{1}^{W}\left(\phi_{k}\right)\right),
$$

whenever $p_{1}+\cdots+p_{m}=p$. Let $H_{f}$ denote the Hilbert space in $L^{2}(S)$ spanned by all functions

$$
s \rightarrow \int_{S^{p-1}} f\left(x_{1}, \ldots, x_{p-1}, s\right) h\left(x_{1}, \ldots, x_{p-1}\right) m\left(d x_{1}\right) \cdots m\left(d x_{p-1}\right)
$$

where $h \in L^{2}\left(S^{p-1}\right)$, and similarly define $H_{g}$. Condition $\left\|f \otimes_{1} g\right\|_{L^{2}\left(S^{p+q-2}\right)}^{2}=0$ implies that $H_{f}$ and $H_{g}$ are orthogonal. Let $\left\{\phi_{n}\right\}$ be an orthonormal basis for $H_{f}$ and let $\left\{\psi_{n}\right\}$ be an orthonormal basis for $H_{g}$. Clearly the Gaussian sequences $\left\{I_{1}^{W}\left(\phi_{n}\right)\right\}$ and $\left\{I_{1}^{W}\left(\psi_{n}\right)\right\}$ are uncorrelated, thus independent. Since $f$ and $g$ admit orthogonal expansions in the tensor product bases,

$$
\begin{aligned}
& f=\sum a_{i_{1}, \ldots, i_{p}} \phi_{i_{1}} \otimes \cdots \otimes \phi_{i_{p}}, \\
& g=\sum b_{j_{1}, \ldots, j_{q}} \psi_{j_{1}} \otimes \cdots \otimes \psi_{j_{q}},
\end{aligned}
$$

the relation (5.5) yields independence of $I_{p}^{W}(f)$ and $I_{q}^{W}(g)$.

From the proof of Theorem 5.1 we obtain an interesting criterion for independence of multiple Itô-Wiener integrals (not included in Üstünel and Zakai (1989)). Corollary 5.2. $\operatorname{Cov}\left(I_{p}^{W}(f)^{2}, I_{q}^{W}(g)^{2}\right) \geq 0$, for any $f \in L^{2}\left(S^{p}\right)$ and $g \in L^{2}\left(S^{q}\right)$. Moreover, $I_{p_{1}}^{W}\left(f_{1}\right), \ldots, I_{p_{n}}^{W}\left(f_{n}\right)$ are independent if and only if their squares $\left(I_{p_{1}}^{W}\left(f_{1}\right)\right)^{2}, \ldots,\left(I_{p_{n}}^{W}\left(f_{n}\right)\right)^{2}$ are uncorrelated. Here $f_{i} \in L^{2}\left(S^{p_{i}}\right), i=1, \ldots, n$.

Proof. The first claim follows from (5.3)-(5.4). If $\operatorname{Cov}\left(I_{p_{i}}^{W}\left(f_{i}\right)^{2}, I_{p_{j}}^{W}\left(f_{j}\right)^{2}\right)=0$ then, again from (5.3)-(5.4), $\left\|f_{i} \otimes_{1} f_{j}\right\|_{L^{2}\left(S^{p_{i}+p_{j}-2}\right)}^{2}=0$. Then the Hilbert spaces $H_{f_{i}}$ and $H_{f_{j}}$ defined as in the proof of the sufficiency part in Theorem 5.1, are orthogonal. This yields the independence of $I_{p_{1}}^{W}\left(f_{n}\right), \ldots, I_{p_{n}}^{W}\left(f_{n}\right)$ in the same way as above. 
It is interesting to compare Corollary 5.2 with the basic fact that jointly normal random variables are independent if and only if they are uncorrelated. Such statement is false for multiple Itô-Wiener integrals because multiple integrals of different orders are always uncorrelated. However, the corollary shows that zero covariance between the squares of integrals suffices for the independence.

Finally we will show that $\operatorname{Cov}\left(I_{p}^{N}(f)^{2}, I_{q}^{N}(g)^{2}\right)=0$ does not imply independence of multiple integrals $I_{p}^{N}(f)$ and $I_{q}^{N}(g)$ with respect to a centered Poisson random measure $N$. This will give a counterexample to the conclusion of the proof of Theorem 1 in Privault (1996). Therefore, necessary and sufficient conditions for independence of multiple Poisson integrals have not been established as far as we know. We expect them to be the same as in Theorem 4.3.

Example 5.3. Let $S=[-1,1], m$ the Lebesgue measure and let $N$ be the centered Poisson random measure on $[-1,1]$, i.e., $E \exp i \theta N(A)=\exp \left[m(A)\left(e^{i \theta}-\right.\right.$ $1-i \theta)]$. Let $f:[-1,1]^{2} \rightarrow \mathbf{R}$ be given by

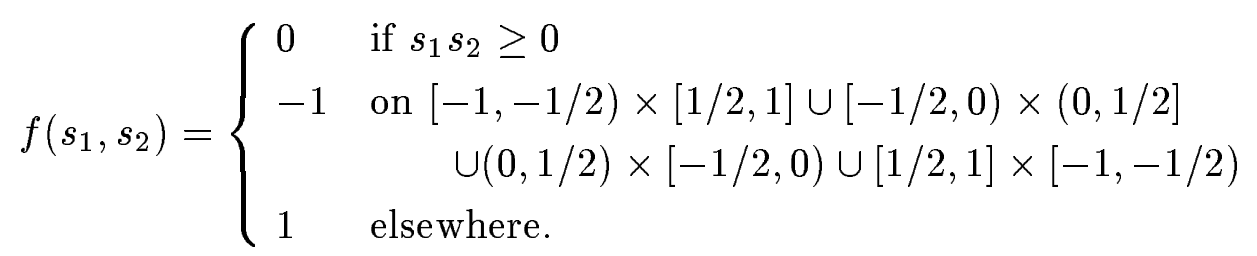

Define $g:[-1,1] \rightarrow \mathbf{R}$ by

$$
g(s)= \begin{cases}-1 & \text { if } s<0 \\ 1 & \text { if } s \geq 0\end{cases}
$$

Therefore we have $p=2$ and $q=1$. Elementary but tedious computations give $E\left[I_{2}^{N}(f)^{2} I_{1}^{N}(g)^{2}\right]=8, E\left[I_{2}^{N}(f)^{2}\right]=4$, and $E\left[I_{1}^{N}(g)^{2}\right]=2$. Consequently, $\operatorname{Cov}\left(I_{2}^{N}(f)^{2}, I_{1}^{N}(g)^{2}\right)=0$. To prove that $I_{2}^{N}(f)$ and $I_{1}^{N}(g)$ are not independent we notice that $I_{2}^{N}(f)$ and $I_{1}^{N}(g)$ take on only integer values. Moreover, if $I_{2}^{N}(f)=2$ then $I_{1}^{N}(g)$ must be even. Since the events $\left\{I_{2}^{N}(f)=2\right\}$ and $\left\{I_{1}^{N}(g)=1\right\}$ have positive probabilities, $I_{2}^{N}(f)$ and $I_{1}^{N}(g)$ are dependent. 
INDEPENDENCE OF MULTIPLE INTEGRALS

\section{REFERENCES}

de la Pena, V.H. and Montgomery-Smith, S.J. (1995), Decoupling inequalities for the tail probabilities of multivariate $U$-statistics, Annals of Probability 23, 817-851.

Kallenberg, O. (1991), On an independence criterion for multiple Wiener integrals, Annals of Probability 19, 483-485.

Kallenberg, O. and Szulga, J. (1989), Multiple integration with respect to Poisson and Lévy processes, Probability Theory and Related Fields 83, 101-134.

Kwapień, S. and Woyczyński, W. (1992), Random Series and Stochastic Integrals: Single and Multiple, Birkhäuser.

Ledoux, M. and Talagrand, M. (1991), Probability in Banach Spaces: Isoperimetry and Processes, Springer Verlag.

Le Page, R. (1980), Multidimentional infinitely divisible variables and processes, Part 2 , Lectures Notes in Mathematics 860, Springer-Verlag, 279-284.

Major, P. (1980), Multiple Wiener-Itô integral, Lecture Notes in Mathematics 849, SpringerVerlag.

Privault, N. (1996), On the independence of multiple stochastic integrals with respect to a class of martingales, C. R. Acad. Sci. Paris 323, 515-520.

Rajput, B. and Rosiński, J. (1989), Spectral representations of infinitely divisible processes, Probability Theory and Related Fields 82, 451-458.

Rosiński, J. (1990), On series representations of infinitely divisible random vectors, Annals of Probability 18, 405-430.

Samorodnitsky, G. and Szulga, J. (1989), An asymptotic evaluation of the tail of a multiple symmetric $\alpha$-stable integral, Annals of Probability 17, 1503-1520.

Samorodnitsky, G. and Taqqu, M.S. (1990), Multiple stable integrals of Banach-valued functions, Journal of Theoretical Probability 3, 267-287.

Samorodnitsky, G. and Taqqu, M.S. (1994), Non-Gaussian Stable Processes, Chapman \& Hall.

Urbanik, K. (1967), Some prediction problems for strictly stationary processes, Proceedings of the Fifth Berkeley Symposium 2, 235-258.

Üstünel, A.S. and Zakai, M. (1989), On the independence and conditioning on Wiener space, Annals of Probability 17, 1441-1453.

Jan Rosiński, University of Tennessee, Department of Mathematics, Ayres Hall, KNOXVILle, TN 37996 USA

E-mail address: rosinski@math.utk.edu

Gennady Samorodnitsky, Cornell University, School of Operations Research and Industrial ENGineERING, ETC BuILding, IthaCA, NY 14853 USA

E-mail address: gennady@orie.cornell.edu 